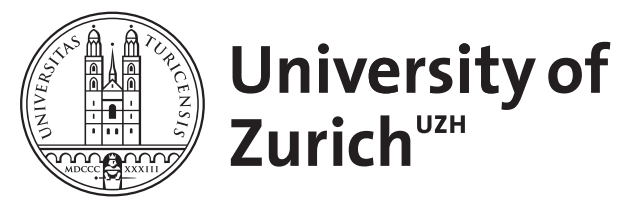

\title{
Saturation estimates for hp-finite element methods
}

Bank, Randolph E ; Parsania, Asieh ; Sauter, Stefan

\begin{abstract}
In this paper we will prove saturation estimates for the adaptive (Formula presented.)-finite element method for linear, second order partial differential equations. More specifically we will consider a sequence of nested finite element discretizations where we allow for both, local mesh refinement and locally increasing the polynomial order. We will prove that the energy norm of the error on the finer level can be estimated by the sum of a contraction of the old error and data oscillations. We will derive estimates of the contraction factor which are explicit with respect to the local mesh width and the local polynomial degree. In order to cover (Formula presented.)-refinement of finite element spaces new polynomial projection operators will be introduced and new polynomial inverse estimates will be derived.
\end{abstract}

DOI: https://doi.org/10.1007/s00791-015-0234-2

Posted at the Zurich Open Repository and Archive, University of Zurich

ZORA URL: https://doi.org/10.5167/uzh-118593

Journal Article

Accepted Version

Originally published at:

Bank, Randolph E; Parsania, Asieh; Sauter, Stefan (2013). Saturation estimates for hp-finite element methods. Computing and Visualization in Science, 16(5):195-217.

DOI: https://doi.org/10.1007/s00791-015-0234-2 


\title{
Saturation Estimates for $h p$-Finite Element Methods*
}

\author{
R. Bank ${ }^{\dagger}$ \\ A. Parsania \\ S. Sauter ${ }^{\S}$ \\ Dedicated to Dietrich Braess on the occasion of his 75th birthday \\ and Wolfgang Hackbusch on the occasion of his 65th birthday
}

\begin{abstract}
In this paper we will prove saturation estimates for the adaptive $h p$-finite element method for linear, second order partial differential equations. More specifically we will consider a sequence of nested finite element discretizations where we allow for both, local mesh refinement and locally increasing the polynomial order. We will prove that the energy norm of the error on the finer level can be estimated by the sum of a contraction of the old error and data oscillations. We will derive estimates of the contraction factor which are explicit with respect to the local mesh width and the local polynomial degree. In order to cover $p$-refinement of finite element spaces new polynomial projection operators will be introduced and new polynomial inverse estimates will be derived.
\end{abstract}

Keywords: $h p$ finite elements, saturation estimate, a posteriori error estimate, linear elliptic equation, polynomial inverse estimates.

Mathematics Subject Classification (2000): 65N15, 65N30,65N35, 65N50

\section{Introduction}

In this paper, we will consider the discretization of linear, second order elliptic partial differential equations by finite elements. Nowadays, adaptive techniques based on a posteriori error estimation have been established to set up a sequence of finite element approximations which should converge towards the exact solution. The advantage compared to uniform mesh refinement is that the finite element spaces are enriched from level to level in a problem oriented way.

A posteriori error estimation and adaptivity are well established methodologies for the numerical solution of partial differential equations by finite elements (cf. [2], [3], [22], [1], [4], [19], [10], [14], [21], [7]).

\footnotetext{
${ }^{*}$ Part of this work has been carried out during a visit of the third author at the Department of Mathematics, University of California, San Diego, La Jolla. This support is gratefully acknowledged.

$\dagger$ Department of Mathematics University of California, San Diego, La Jolla, California 92093-0112, USA, e-mail: rbank@ucsd.edu

${ }^{\ddagger}$ Institut für Mathematik, Universität Zürich, Winterthurerstrasse 190, CH-8057 Zürich, Switzerland, email: asieh.parsania@math.uzh.ch

§Institut für Mathematik, Universität Zürich, Winterthurerstrasse 190, CH-8057 Zürich, Switzerland, email: stas@math.uzh.ch
} 
Some types of error estimators as, e.g., hierarchical error estimators (see, e.g., [5], [8], [6]) require explicitly or implicitly the saturation assumption which states that the error on the refined mesh and/or with higher polynomial degree is strictly smaller than the error on the previous mesh/polynomial degree. In the pioneering paper [11] the saturation assumption is proved for the $\mathbb{P}_{1}$-finite element method for the Poisson problem in two spatial dimensions under the assumption that the data oscillations are small. In [14] the convergence of adaptive finite element methods (AFEM) for general (nonsymmetric) second order linear elliptic partial differential equations is proved, where the term "adaptivity" is understood in the sense of adaptive mesh refinement and the polynomial degree stays fixed. The theory in [14] also generalizes the proof of the saturation property to quite general 2nd order elliptic problems and estimate the error on the refined mesh by the error of the coarser mesh plus a data oscillation term.

In this paper, we will focus on adaptive $h p$-refinement, i.e., the finite element space is enriched by increasing locally the polynomial degree of the ansatz functions while we allow also for conventional local $h$-refinement, where the elements of the finite element mesh are geometrically subdivided. We will show (and quantify) that, for residual a posteriori error estimation, the saturation property, i.e., the error contraction from level to level behaves like $\left(1-\frac{C}{p^{5 / 2}}\right)$ provided the data oscillations are sufficiently well resolved. Hence, $p$-refinement should be combined with $h$-refinement in order to guarantee that the numerical solution converges towards the exact solution.

The paper is organised as follows. In Section 2 we will introduce the elliptic boundary value problem and formulate appropriate assumptions to ensure the well-posedness of this problem.

The $h p$-finite element method will be defined in Section 3 and standard assumptions on mesh refinement, shape regularity, and the polynomial degree distribution will be introduced.

In Section 4 we will recall the definition of the residual a posteriori error estimator for $h p$-finite elements and its reliability estimate.

In Section 5 we will introduce some polynomial projection operator which maps global polynomials on triangle patches to piecewise polynomials of lower degree. This allows to localize projected residuals by multiplying the resulting piecewise polynomials with appropriate bubble functions. We will investigate the stability constant of the projection operator while its explicit dependence on the polynomial degree for $p$-refinement will be analysed numerically in Appendix A.

The saturation estimate will be proved in Section 6 .

In Appendix B we will derive polynomial inverse estimates containing those bubble functions as weights which have been used in Section 6 to prove the saturation property.

Remark 1.1 The theory in [14] indicates how an adaptive finite element procedure should be defined such that the sequence of finite element solutions converges. Note that the rate of convergence for adaptive finite elements is investigated in, e.g., [7], [21], [20].

Besides the estimates derived for the saturation property, the convergence theory requires a reduction of the data oscillations which, for h-refinement, is (essentially) related to the fact that the local mesh width shrinks by a fixed factor for the marked elements. For p-refinement, the analogue condition is that the hp-weight of the data oscillations term also shrinks by a factor smaller than one. Due to the non-robust p-dependence of polynomial inverse estimates this cannot be expected in a straightforward way. In order not to overload this paper we decided to leave the convergence of an adaptive hp-finite element method to a forthcoming paper. 


\section{Setting}

Let $\Omega \subset \mathbb{R}^{d}$ be a bounded Lipschitz domain. Consider the Dirichlet problem for given $f \in$ $L^{2}(\Omega)$ :

$$
\begin{aligned}
-\operatorname{div}(\mathbf{A} \nabla u)+\langle\mathbf{b}, \nabla u\rangle+c u & =f & & \text { in } \Omega, \\
u & =0 & & \text { on } \partial \Omega
\end{aligned}
$$

with variational formulation: Find $u \in H_{0}^{1}(\Omega)$ such that

$$
a(u, v):=\int_{\Omega}\langle\mathbf{A} \nabla u, \nabla v\rangle+(\langle\mathbf{b}, \nabla u\rangle+c u) v=\int_{\Omega} f v=: F(v) \quad \forall v \in H_{0}^{1}(\Omega) .
$$

Assumption 2.1 The coefficients in (2.2) satisfy $\mathbf{A} \in C^{0,1}\left(\Omega, \mathbb{R}_{\mathrm{sym}}^{d \times d}\right), \mathbf{b} \in C^{0,1}\left(\Omega, \mathbb{R}^{d}\right)$, $c \in L^{\infty}(\Omega)$, and

$$
\begin{aligned}
& 0<\alpha:=\inf _{x \in \Omega} \inf _{v \in \mathbb{R}^{d} \backslash\{0\}} \frac{\langle\mathbf{A} v, v\rangle}{\langle v, v\rangle} \leq \sup _{x \in \Omega} \sup _{v \in \mathbb{R}^{d} \backslash\{0\}} \frac{\langle\mathbf{A} v, v\rangle}{\langle v, v\rangle}:=\beta<\infty \\
& 0 \leq \inf _{x \in \Omega}\left(c(x)-\frac{1}{2} \operatorname{div} \mathbf{b}(x)\right) .
\end{aligned}
$$

We set $c_{\infty}:=\|c\|_{L^{\infty}(\Omega)}$ and $b_{\infty}:=\max \left\{\|\mathbf{b}\|_{L^{\infty}\left(\Omega, \mathbb{R}^{d}\right)},\|\operatorname{div} \mathbf{b}\|_{L^{\infty}(\Omega)}\right\}$. The energy norm is denoted by

$$
\|v\|_{\mathrm{PDE}}:=a(v, v)^{1 / 2}
$$

where Assumption 2.1 implies that $\|\cdot\|_{\mathrm{PDE}}$ is a norm and Friedrichs inequality implies

$$
\|v\|_{\mathrm{PDE}}^{2} \geq \int_{\Omega}\langle\mathbf{A} \nabla v, \nabla v\rangle \geq \alpha\|\nabla v\|_{L^{2}(\Omega)}^{2} \geq \frac{\alpha}{c_{\mathrm{F}}}\|v\|_{H^{1}(\Omega)}^{2}
$$

where $c_{\mathrm{F}}$ denotes the Friedrichs constant. In fact, the norms $\|\cdot\|_{\mathrm{PDE}}$ and $\|\cdot\|_{H^{1}(\Omega)}$ are equivalent since also

$$
\|v\|_{\mathrm{PDE}}^{2} \leq C_{a}\|v\|_{H^{1}(\Omega)}^{2} \quad \text { with } \quad C_{a}:=\frac{b_{\infty}}{2}+\max \left\{c_{\infty}, \beta\right\}
$$

For a subdomain $\omega \subset \Omega$ we set

$$
\|v\|_{\mathrm{PDE}, \omega}^{2}:=a_{\omega}(v, v):=\int_{\omega}\left(\langle\mathbf{A} \nabla v, \nabla v\rangle+\langle\mathbf{b}, \nabla v\rangle v+c v^{2}\right) .
$$

Remark 2.2 The constants in the estimates below possibly depend (continuously) on $\alpha, \beta, c_{\infty}$, and $b_{\infty}$ and might tend to infinity with increasing $\beta, b_{\infty}, c_{\infty}, \alpha^{-1}$. We suppress the dependence in the notation.

Note that these conditions ensure that problem (2.2) is well posed and the coercivity estimate holds trivially

$$
a(v, v)=\|v\|_{\mathrm{PDE}}^{2} \quad \forall v \in H_{0}^{1}(\Omega) .
$$

Assumption 2.1 implies the continuity of $a(\cdot, \cdot)$, i.e.,

$$
a(u, v) \leq C_{S}\|v\|_{\mathrm{PDE}}\|v\|_{\mathrm{PDE}} \quad \forall v \in H_{0}^{1}(\Omega)
$$

with $C_{S}:=1+c_{\mathrm{F}} \frac{b_{\infty}}{\alpha}$. 


\section{Conforming hp-Finite Elements}

Let $\Omega \subset \mathbb{R}^{2}$ be a polygonal domain and let $\mathcal{T}:=\left\{K_{i}: 1 \leq i \leq N\right\}$ denote a conforming simplicial finite element mesh. With each element $K \in \mathcal{T}$ we associate of polynomial degree $p_{K} \in \mathbb{N}_{\geq 1}$ which are collected into the polynomial degree vector $\mathbf{p}=\left(p_{K}\right)_{K \in \mathcal{T}}$. Then, we define the conforming $h p$-finite element space for the mesh $\mathcal{T}$ with local polynomials of degree $p_{K}$ by

$$
S_{\mathcal{T}}^{\mathbf{p}}:=\left\{u \in H_{0}^{1}(\Omega)|\forall K \in \mathcal{T} \quad u|_{K} \in \mathbb{P}_{p_{K}}\right\}
$$

Here $\mathbb{P}_{p}$ denote the space of bivariate polynomials of maximal total degree $p$. For a subset $\omega \subset \Omega$, we write $\mathbb{P}_{p}(\omega)$ to indicate explicitly that we consider $u \in \mathbb{P}_{p}(\omega)$ as a polynomial on $\omega$. Formally we define $\mathbb{P}_{-1}:=\{0\}$. We set

$$
p_{\mathcal{T}}:=\max \left\{p_{K}: K \in \mathcal{T}\right\} .
$$

By convention the triangles $K \in \mathcal{T}$ are open sets. The boundaries of the triangles $K \in \mathcal{T}$ consist of one-dimensional edges which are collected in the set $\mathcal{E}$. Furthermore, let $\mathcal{E}_{\Omega}:=$ $\{E \in \mathcal{E} \mid E \subset \Omega\}$. The union $\mathfrak{S}_{\Omega}:=\bigcup_{E \in \mathcal{E}_{\Omega}} E$ forms the inner skeleton of the mesh $\mathcal{T}$. For each $E \in \mathcal{E}$ we fix one unit vector $\mathbf{n}_{E}$ which is perpendicular to $E$. If $E \subset \partial \Omega$, the orientation is chosen such that $\mathbf{n}_{E}$ points to the exterior of $\Omega$. The $\mathcal{E}$-piecewise constant vector field $\mathbf{n}$ is given by $\left.\mathbf{n}\right|_{E}:=\mathbf{n}_{E}$. Finally we define the jump of some piecewise smooth function $g \in \prod_{K \in \mathcal{T}} H^{1}(K)$ across $E \in \mathcal{E}_{\Omega}$ by

$$
[g]_{E}(x):=\lim _{\varepsilon \searrow 0}\left(g\left(x+\varepsilon \mathbf{n}_{E}\right)-g\left(x-\varepsilon \mathbf{n}_{E}\right)\right) \quad \forall x \in \stackrel{\circ}{E} .
$$

This defines the jump function $\left.[g]\right|_{E}:=[g]_{E}$ for all $E \in \mathcal{E}_{\Omega}$ almost everywhere.

Let $\mathcal{N}_{\Omega}^{1}$ denote the set of inner vertices of $\mathcal{T}$. For $z \in \mathcal{N}_{\Omega}^{1}$, we denote by $b_{z}^{1} \in S_{\mathcal{T}}^{1}$ the canonical continuous, piecewise affine basis function. The volume star for the node $z$ is given by $\omega_{z}:=\operatorname{supp} b_{z}^{1}$ and its measure is denoted by $\left|\omega_{z}\right|$. For $z \in \mathcal{N}_{\Omega}^{1}$, we set $\mathcal{E}_{z}:=$ $\left\{E \in \mathcal{E}: E \subset \mathfrak{S}_{\Omega} \cap \stackrel{\circ}{\omega}_{z}\right\}$ and $\mathcal{T}_{z}:=\left\{K \in \mathcal{T}: K \subset \omega_{z}\right\}$. Let $\mathcal{V}_{K}$ denote the set of inner vertices of $K$ and let $\omega_{K}:=\bigcup_{z \in \mathcal{V}_{K}} \omega_{z}$.

We denote by $\nabla_{\mathcal{T}}$ the trianglewise gradient and by $\operatorname{div}_{\mathcal{T}}$ the trianglewise divergence operator. Let $\mathfrak{h}_{\mathcal{T}}$ denote the $\mathcal{T}$-piecewise constant function with values $\left.\mathfrak{h}_{\mathcal{T}}\right|_{K}:=\operatorname{diam} K$ for all $K \in \mathcal{T}$. Similarly we define $\mathfrak{h}_{\mathcal{E}}: \mathfrak{S}_{\Omega} \rightarrow \mathbb{R}$ as the $\mathcal{E}$-piecewise constant function $\left.\mathfrak{h}_{\mathcal{E}}\right|_{E}:=\operatorname{diam} E$ for all $E \in \mathcal{E}_{\Omega}$. The maximal mesh width in $\mathcal{T}$ is defined by

$$
h_{\mathcal{T}, \max }:=\max \{\operatorname{diam} K: K \in \mathcal{T}\} .
$$

If $\mathcal{T}$ is clear from the context we write $h$ short for $h_{\mathcal{T} \text {,max }}$. The shape regularity of $\mathcal{T}$ is described by the constant

$$
\rho_{\mathcal{T}}:=\max \left\{\frac{\operatorname{diam} K}{\operatorname{diam} \mathcal{B}_{K}}: K \in \mathcal{T}\right\}
$$

where $\mathcal{B}_{K}$ is the maximal inscribed ball in $K$. Since $\mathcal{T}$ contains finitely many simplices the constant $\rho_{\mathcal{T}}$ is always bounded but becomes large if the simplices are degenerate, e.g., are 
flat or needle-shaped. The constants in the following estimates depend on the mesh via the constant $\rho_{\mathcal{T}}$ - they are bounded for any fixed $\rho_{\mathcal{T}}$ but, possibly, become large for large $\rho_{\mathcal{T}}$.

Concerning the polynomial degree distribution we assume throughout the paper that the polynomial degrees of neighbouring elements are comparable ${ }^{1}$ :

$$
\rho_{\mathcal{T}}^{-1}\left(p_{K}+1\right) \leq p_{K^{\prime}}+1 \leq \rho_{\mathcal{T}}\left(p_{K}+1\right) \quad \forall K, K^{\prime} \in \mathcal{T} \text { with } \bar{K} \cap \overline{K^{\prime}} \neq \emptyset .
$$

The finite element solution is defined by:

$$
\text { Find } u_{\mathcal{T}}^{\mathbf{p}} \in S_{\mathcal{T}}^{\mathbf{p}} \quad \text { such that } a\left(u_{\mathcal{T}}^{\mathbf{p}}, v\right)=F(v) \quad \forall v \in S_{\mathcal{T}}^{\mathbf{p}} \text {. }
$$

In view of an adaptive solution process we generate a sequence $S_{\ell}:=S_{\mathcal{T}_{\ell}}^{\mathbf{p}_{\ell}}, \ell \in \mathbb{N}_{0}$, of finite element spaces, where we require that all meshes $\mathcal{T}_{\ell}$ are conforming and the constants $\rho_{\ell}$ corresponding to the shape regularity of the mesh $\mathcal{T}_{\ell}$ and the polynomial degree vector $\mathbf{p}_{\ell}$ are uniformly bounded from above by some positive constant $\rho$. We also assume that $\mathcal{T}_{\ell+1}$ is a refinement of $\mathcal{T}_{\ell}$ in the sense that for any $K \in \mathcal{T}_{\ell}$ there is a subset sons $(K) \subset \mathcal{T}_{\ell+1}$ such that

$$
\bar{K}=\bigcup_{K^{\prime} \in \operatorname{sons}(K)} \overline{K^{\prime}}
$$

To reduce technicalities we make the following assumption concerning the concrete refinement method (cf. Figure 1). As usual for conforming $h$-refinement, there exists two types of refinements. Some triangles are marked for refinement while this marking induces some additional refinement of neighbouring triangles in order to avoid hanging nodes.

\section{Assumption 3.1}

a. A triangle $K$, which is marked for refinement, is regularly refined by connecting the midpoint of the edges as well as the midpoint of the longest side with the opposite vertex in $K$ (cf. Fig. 1, Pic 1) so that the set sons $(K)$ contains six new triangles.

b. To eliminate hanging nodes neighbouring triangles are refined by inserting a line $L$ from one hanging node to the opposite vertex and connecting the vertices of $K$ with the midpoint of L (cf. Fig. 1, Pic 2). If there is a further hanging node then this node is connected also with the midpoint of $L$ (cf. Fig. 1, Pic 3). If $K$ contains three hanging nodes or the shape regularity of the new triangles exceeds some threshold it will be regularly refined.

c. For any triangle $K \in \mathcal{T}_{\ell}$, one of the following conditions are satisfied (cf. Fig. 2):

i. $K$ will be $p$-refined, i.e., $K \in \mathcal{T}_{\ell+1}$ and the polynomial degree is raised by 1.

ii $K$ will be h-refined, i.e., there exists a set of sons $\sigma(K) \subset \mathcal{T}_{\ell+1}$ with $\bar{K}=\bigcup_{K^{\prime} \in \operatorname{sons}(K)} \overline{K^{\prime}}$ and at least one vertex of each $K^{\prime}$ lies in the interior of $K$. The polynomial degree $p_{K}$ defines the polynomial degree on $K^{\prime} \in$ sons $(K)$ as follows

$$
p_{K^{\prime}}:=\left\{\begin{array}{l}
p_{K} \quad \text { if } K \text { is regularly refined, } \\
p_{K}+1 \quad \text { if }\left(\begin{array}{l}
\exists \widetilde{K} \in \mathcal{T}_{\ell}: \overline{K^{\prime}} \cap \widetilde{\widetilde{K}} \text { is a full edge of } \widetilde{K} \\
\widetilde{K} \text { is } p-\text { refined } \\
p_{\widetilde{K}} \geq p_{K}
\end{array}\right), \\
p_{K} \quad \text { otherwise. }
\end{array}\right.
$$

\footnotetext{
${ }^{1}$ We use here the same constant $\rho$ as for the shape regularity to simplify the notation.
} 


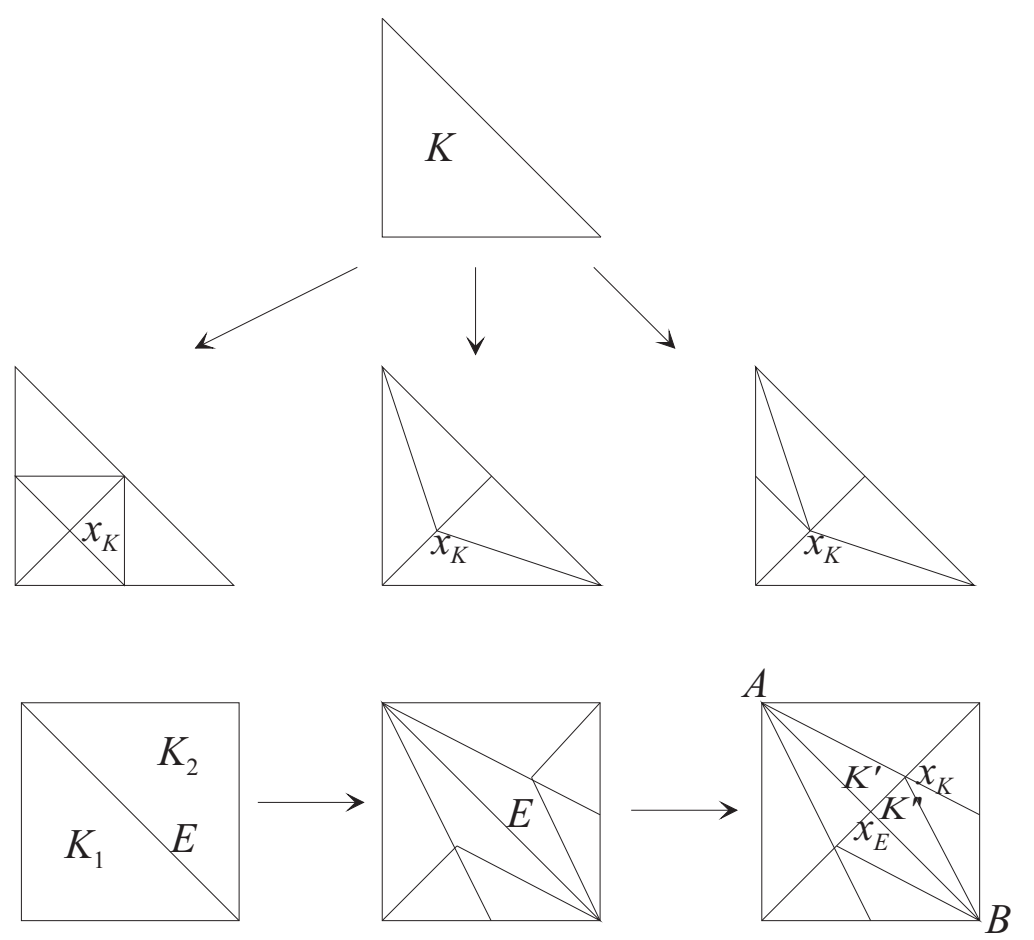

Figure 1: Refinement patterns of a triangle which satisfies the interior node property. Second row, from left to right: Pic. 1: Regular refinement. Pic. 2,3: Refinement patterns for the elimination of hanging nodes. Third row: If two triangles $K_{1}, K_{2}$ share an edge $E$ and they will be both $h$-refined, then the common edge $E$ must get an interior point $x_{E}$.

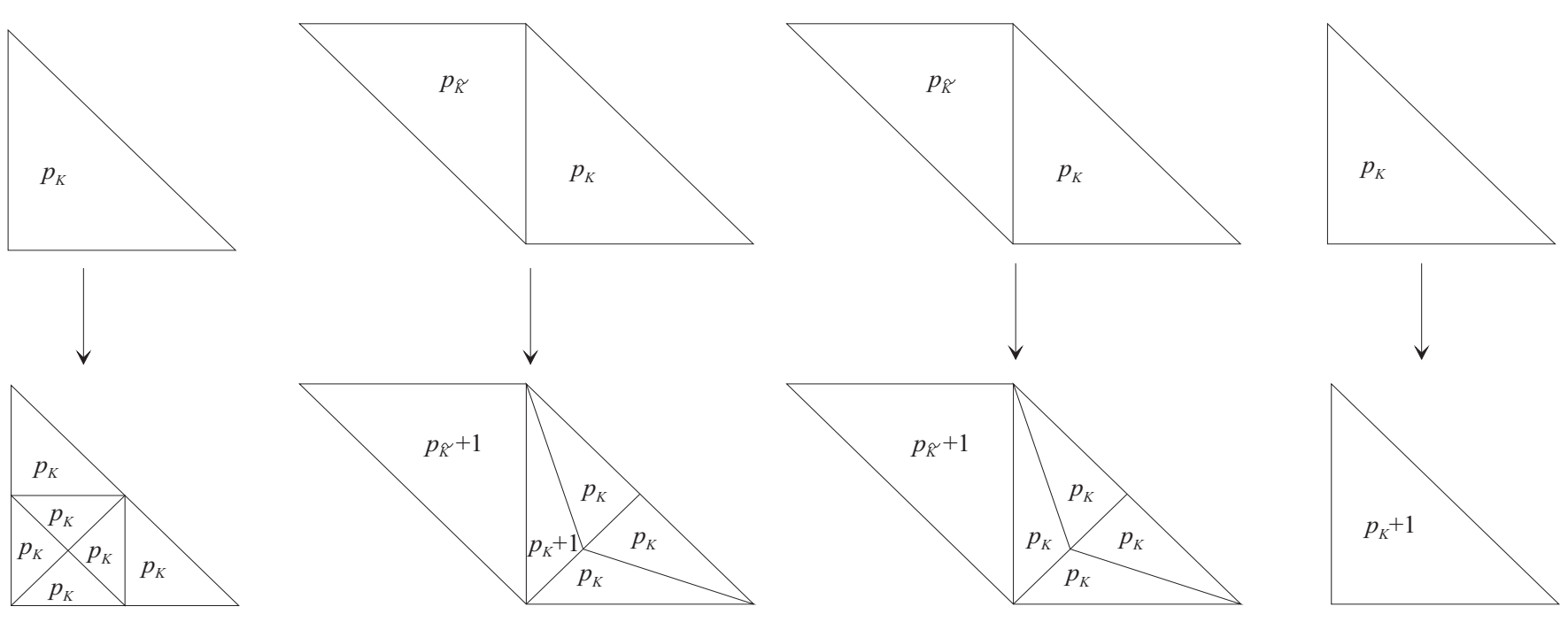

Figure 2: Definition of the polynomial degrees. From left to right: Pic1: regular refinement. Pic 2: $K$ is $h$-refined, $\widetilde{K}$ is $p$-refined and $p_{\widetilde{K}} \geq p_{K}$. Pic $3: K$ is $h$-refined, $\widetilde{K}$ is $p$-refined and $p_{\widetilde{K}}<p_{K}$. Pic 4: $p$-refinement. 
Assumption 3.1 implies the interior node property (cf. [14, Sec. 3.4]).

Definition 3.2 (interior node property) Any $K \in \mathcal{T}_{\ell}$ which will be regularly $h$-refined and the three adjacent triangles $T^{\prime} \in \mathcal{T}_{\ell}$ as well as their common sides contain a node of the finer mesh $\mathcal{T}_{\ell+1}$ in their interior and the resulting triangulation $\mathcal{T}_{\ell+1}$ has no hanging nodes.

Remark 3.3 Let $K_{1}, K_{2} \in \mathcal{T}_{\ell}$ denote two triangles which share an edge $E$ and let $p_{m}:=p_{K_{m}}$, $m=1,2$. The condition $u \in H_{0}^{1}(\Omega)$ in the definition of $S_{\mathcal{T}_{\ell}}^{\mathbf{p}_{\ell}}$ in (3.1) implies that for any $u \in S_{\ell}$ the one-dimensional polynomial degree of $u$ along $E$ satisfies

$$
\operatorname{deg}\left(\left.u\right|_{E}\right) \leq p_{E}:=\min \left\{p_{1}, p_{2}\right\} .
$$

Notation 3.4 To reduce the number of indices we write $u_{\ell}$ short for $u_{\mathcal{T}_{\ell}}^{\mathbf{p}_{\ell}}, \mathfrak{h}_{\ell}$ short for $\mathfrak{h}_{\mathcal{T}_{\ell}}$, $\operatorname{div}_{\ell}$ short for $\operatorname{div}_{\mathcal{T}_{\ell}}, p_{\ell}$ short for $p_{\mathcal{T}_{\ell}}, \mathcal{N}_{\ell}^{1}$ for the inner triangle vertices for the mesh $\mathcal{T}_{\ell}$, etc. The star $\omega_{z}$ corresponds always to the triangulation $\mathcal{T}_{\ell}$ while we suppress this additional index in the notation of $\omega_{z}$.

Definition 3.5 The saturation estimate for a sequence of finite element solutions $\left(u_{\ell}\right)_{\ell}$ is an estimate of the form

$$
\left\|u_{\ell+1}-u\right\|_{\mathrm{PDE}} \leq \kappa_{\ell}\left\|u_{\ell}-u\right\|_{\mathrm{PDE}}
$$

for some $\kappa_{\ell}<1$ such that $\prod_{\ell=1}^{\infty} \kappa_{\ell}=0$.

It was proved in [11] that the saturation estimate holds for the case of a bounded, twodimensional domain $\Omega$ with coefficients

$$
\mathbf{A}=\mathbf{I}, \quad \mathbf{b}=\mathbf{0} \quad \text { and } \quad c=0,
$$

where $\mathbf{I}$ is the $2 \times 2$ unit matrix and the analysis was restricted to $\mathbb{P}_{1}$ finite elements with $h$-refinement. It was proved that it is necessary and sufficient for the saturation estimate that the data oscillations (which will be introduced in (6.5)) are controlled. Here, we generalize this result to the setting described in Section 2 and also derive $p$-explicit estimates for the contraction factor $\kappa_{\ell}$.

\section{Residual A Posteriori Error Estimation}

The Galerkin error is denoted by $e_{\ell}:=u-u_{\ell}$. In the following, we will investigate under which condition the saturation estimate of the form

$$
\left\|e_{\ell+1}\right\|_{\mathrm{PDE}} \leq \kappa_{\ell}\left\|e_{\ell}\right\|_{\mathrm{PDE}}
$$

hold for some $\left.\kappa_{\ell} \in\right] 0,1[$ depending only on the polynomial degree $p$ and the shape-regularity of the mesh but not on the mesh width.

For the proof of the saturation estimate, we will use tools from residual a posteriori error estimation which we briefly recall: To obtain an a posteriori error estimate we obtain by Galerkin's orthogonality for every $v \in S_{\ell}$

$$
\left\|e_{\ell}\right\|_{\mathrm{PDE}}^{2}=a\left(e_{\ell}, e_{\ell}-v\right)=\int_{\Omega} \operatorname{res}\left(u_{\ell}\right)\left(e_{\ell}-v\right)+\int_{\mathfrak{S}_{\Omega}} \operatorname{Res}\left(u_{\ell}\right)\left(e_{\ell}-v\right),
$$


where the volume residual res : $S_{\ell} \rightarrow L^{2}(\Omega)$ is given by

$$
\operatorname{res}(v):=f+\operatorname{div}_{\ell}(\mathbf{A} \nabla v)-\langle\mathbf{b}, \nabla v\rangle-c v
$$

and the edge residual Res $: S_{\ell} \rightarrow L^{2}\left(\mathfrak{S}_{\Omega}\right)$ is given by

$$
\operatorname{Res}(v):=-\langle\mathbf{A n},[\nabla v]\rangle \quad \text { a.e. in } \mathfrak{S}_{\Omega} \text {. }
$$

By choosing $v \in S_{\ell}$ as the Clément interpolation of $e_{\ell}$ and using a trace inequality for the last term in (4.2), results in the classical residual a posteriori error estimation. In [15], [16] the local and global residual a posteriori error estimator is defined by

$$
\eta_{K}^{2}(v):=\left\|\frac{h_{K}}{p_{K}} \operatorname{res}(v)\right\|_{L^{2}(K)}^{2}+\sum_{E \subset \partial K \cap \Omega}\left\|\sqrt{\frac{h_{E}}{2 p_{K}}} \operatorname{Res}(v)\right\|_{L^{2}(E)}^{2} \quad \forall v \in S_{\ell} \quad \forall K \in \mathcal{T}_{\ell} .
$$

The global error estimator is given by

$$
\eta_{\ell}(v):=\sqrt{\sum_{K \in \mathcal{T}_{\ell}} \eta_{K}^{2}(v)}
$$

Due to the finite overlap of the stars $\omega_{z}$, the error estimator (4.3) is equivalent to

$$
\eta_{\ell}^{\mathrm{star}}(v):=\sqrt{\sum_{z \in \mathcal{N}_{\ell}^{1}} \eta_{z}^{2}(v)} \quad \text { with } \quad \eta_{z}^{2}(v):=\left\|\frac{h_{z}}{p_{z}} \operatorname{res}(v)\right\|_{L^{2}\left(\omega_{z}\right)}^{2}+\sum_{E \in \mathcal{E}_{z}}\left\|\sqrt{\frac{h_{z}}{p_{z}}} \operatorname{Res}(v)\right\|_{L^{2}(E)}^{2}
$$

and

$$
p_{z}:=\min \left\{p_{K}: K \subset \omega_{z}\right\} \quad \text { and } \quad h_{z}:=\max \left\{h_{K}: K \subset \omega_{z}\right\} .
$$

Theorem 4.1 (Melenk, Wohlmuth) Let $\Omega \subset \mathbb{R}^{2}$ be a bounded Lipschitz domain. Let $a(\cdot, \cdot)$ in (2.2) satisfy Assumption 2.1 and let $f \in L^{2}(\Omega)$. The solution of (2.2) is denoted by $u$ and its Galerkin approximation by $u_{\ell}\left(\right.$ see (3.4)). There exists a constant $C_{\mathrm{rel}}$ independent of the local mesh width and the local polynomial degree but, possibly, depending on the constants in Assumption 2.1 such that

$$
\left\|u-u_{\ell}\right\|_{\mathrm{PDE}} \leq C_{\mathrm{rel}} \eta_{\ell}\left(u_{\ell}\right) \leq C_{\mathrm{rel}} \eta_{\ell}^{\mathrm{star}}\left(u_{\ell}\right)
$$

The proof of this theorem is a slight modification of [16, Theorem 3.6] and we include it here for completeness.

Proof. The error $u-u_{\ell}$ can be estimated by using (4.2) and by setting $w=e_{\ell}-I e_{\ell}$ with the $h p$-Clément interpolation operator $I$ as in [16, Section 2.1]:

$$
\left\|e_{\ell}\right\|_{\mathrm{PDE}}^{2}=\int_{\Omega} w \operatorname{res}\left(u_{\ell}\right)+\int_{\mathfrak{S}_{\Omega}} w \operatorname{Res}\left(u_{\ell}\right) \leq C_{\mathrm{rel}} \eta_{\ell}\left(u_{\ell}\right)\left\|e_{\ell}\right\|_{\mathrm{PDE}}
$$

for all $v \in S_{\ell}$. Clearly we have

$$
\eta_{\ell}(v) \leq \eta_{\ell}^{\mathrm{star}}(v) \leq C_{\sharp} \eta_{\ell}(v),
$$

where $C_{\sharp}$ depends only on the constant $\rho_{\ell}$ in (3.2) and (3.3). 


\section{Projection of Polynomials onto Piecewise Polynomi- als}

The proof of the saturation estimate is based on estimates of some projection of the volume residual to the space of piecewise polynomials locally on stars $\omega_{z}$. In this section, we will derive stability estimates for this projection.

We start with a result of a weighed $L^{2}$ projection of global polynomials of maximal total degree $p$ to piecewise polynomials of lower degree. The setting is as follows.

Let $z \in \mathbb{R}^{2}$ and let $\mathcal{T}_{z}:=\left\{K_{i}: 1 \leq i \leq q\right\}$ denote a triangle patch around $z$, i.e., $\mathcal{T}_{z}$ is a set of (open) triangles which

- are pairwise disjoint,

- share $z$ as a common vertex.

- For all $1 \leq i \leq q$, the triangles $K_{i-1}$ and $K_{i}$ share one common edge ${ }^{2}$.

Let $^{3} \omega_{z}:=\operatorname{int}\left(\bigcup_{i=1}^{q} \overline{K_{i}}\right)$ and let $\mathfrak{S}:=\omega_{z} \cap \bigcup_{i=1}^{q} \partial K_{i}$ denote the inner mesh skeleton. We denote by $\mathbb{P}_{p}\left(\mathcal{T}_{z}\right)$ the space of piecewise polynomials, i.e.,

$$
\mathbb{P}_{p}\left(\mathcal{T}_{z}\right):=\left\{f: \omega_{z} \backslash \mathfrak{S} \rightarrow \mathbb{R}|\forall 1 \leq i \leq q ; \quad f|_{K_{i}} \in \mathbb{P}_{p}\left(K_{i}\right)\right\}
$$

Next, we will introduce weighted scalar products and associated norms. The weights are defined triangle- and edge-wise and depend whether the triangle will be $h$-refined of $p$-refined.

\section{Definition 5.1}

a. p-refinement.

If $K$ will be p-refined, then, the cubic weight function $\Phi_{K}^{(3)}$ and quadratic edge bubble $\Phi_{E}$ are given, on the reference element $\widehat{K}:=\operatorname{conv}\left(\left(\begin{array}{l}0 \\ 0\end{array}\right),\left(\begin{array}{l}1 \\ 0\end{array}\right),\left(\begin{array}{l}0 \\ 1\end{array}\right)\right)$ and on the reference interval $\widehat{E}:=(0,1)$, by

$$
\Phi_{\widehat{K}}^{(3)}\left(x_{1}, x_{2}\right)=\left(1-x_{1}-x_{2}\right) x_{1} x_{2} \quad \text { and } \quad \Phi_{\widehat{E}}(x)=x(1-x),
$$

while on $K$ and $E$ we set

$$
\Phi_{K}^{(3)}:=\Phi_{\widehat{K}}^{(3)} \circ \Lambda_{K}^{-1} \quad \text { and } \quad \Phi_{E}:=\Phi_{\widehat{E}} \circ \Lambda_{E}^{-1},
$$

where $\Lambda_{K}: \widehat{K} \rightarrow K$ and $\Lambda_{E}: \widehat{E} \rightarrow E$ are affine pullbacks ${ }^{4}$.

b. h-refinement.

The edge bubble $\Phi_{E}$ for h-refinement is the same as for p-refinement.

b1. Let $K$ be regularly refined (cf. Figure 3). Then, $\Phi_{K, K}^{(1)}$ is the piecewise linear function on the submesh sons $(K)$ which has value 1 at $x_{K}$ and value 0 at all other vertices of the refined mesh. Let $E$ denote the edge as indicated in Figure 3 which splits $K$ into the triangles $K_{1}$ and $K_{2}$. Then $\Phi_{K}^{(2)}$ is the product of the barycentric coordinates for the two endpoints of $E$ with respect to the two triangles $K_{1}$ and $K_{2}$.

\footnotetext{
${ }^{2}$ We use here the convention $K_{0}:=K_{q}$. Clearly $q \geq 3$ holds.

${ }^{3}$ For a subset $\omega \subset \mathbb{R}^{2}$, we denote by int $(\omega)$ the open interior of $\omega$.

${ }^{4}$ Note that the scalings compared to the scalings in [23, p.83] differ by fixed constants of order 1.
} 




Figure 3: Illustration for the notation of a regulary $h$-refined triangle $K$. The edge $E=\overline{A M_{A}}$ splits $K$ into the two triangles $K_{1}$ and $K_{2}$. The subtriangle $K_{1}^{\prime}$ has vertices $A, M_{C}, x_{K}$ while the vertices of $K_{2}^{\prime}$ are $A, x_{K}, M_{B}$.

b2. If $K$ is non-regularly h-refined (cf. Figure 1, Pic. 2,3), then the weight function for $K$ is the piecewise linear bubble function $\Phi_{K}^{(1)}$ which interpolates $\Phi_{K}^{(3)}$ at the vertices of the submesh sons $(K)$.

The weight function for a triangle $K$ is

$$
\Phi_{K}:= \begin{cases}\Phi_{K}^{(3)} & \text { if } K \text { will be p-refined, } \\ \Phi_{K}^{(1)} & \text { if } K \text { will be non-regularly } h \text {-refined } \\ \Phi_{K, K}^{(1)}+\Phi_{K}^{(2)} & \text { if } K \text { will be regularly h-refined. }\end{cases}
$$

For $z \in \mathcal{N}_{\Omega}^{1}$, the function $\Phi_{z}: \omega_{z} \rightarrow \mathbb{R}$ is given by

$$
\left.\Phi_{z}\right|_{K}:=\Phi_{K} \quad \forall K \in \omega_{z}
$$

and extended by zero to $\Omega$.

These weight functions induce bilinear forms $(\cdot, \cdot)_{K}$ and $(\cdot, \cdot)_{z}$ via

$$
(u, v)_{K}:=\int_{K} \Phi_{K} u v \quad \text { and } \quad(u, v)_{z}:=\sum_{K \subset \omega_{z}}(u, v)_{K}=\int_{\omega_{z}} \Phi_{z} u v
$$

and a corresponding norm $\|\cdot\|_{z}:=(\cdot, \cdot)_{z}^{1 / 2}$.

Next we define a projection $\Pi_{K}^{p}: \mathbb{P}_{p}(K) \rightarrow \mathbb{P}_{p-1}(K)$ by

$$
\int_{K} \Phi_{K}\left(\Pi_{K}^{p} v\right) w=\int_{K} \Phi_{K} v w \quad \forall w \in \mathbb{P}_{p-1}(K),
$$

where the definition of $\Phi_{K}$ is as in (5.4), i.e., depends on how $K$ will be refined. 
Definition 5.2 For a triangle patch $\mathcal{T}_{z}$, let $p_{z}$ be as in (4.5). The star-wise polynomial projection $\Pi_{z}$ is applied to polynomials $v \in \mathbb{P}_{p_{z}-1}\left(\omega_{z}\right)$ and given by

$$
\left.\left(\Pi_{z} v\right)\right|_{K}:= \begin{cases}\Phi_{K}^{(3)} \Pi_{K}^{p_{z}-1} v & \text { if } K \text { is p-refined, } \\ \Phi_{K}^{(1)} v & \text { if } K \text { is non-regularly h-refined } \\ \Phi_{K, K}^{(1)} v+\Phi_{K} \Pi_{K}^{p_{z}-1} v & \text { if } K \text { is regularly h-refined, }\end{cases}
$$

Theorem 5.3 Let $p \geq 1$. For all $u \in \mathbb{P}_{p}\left(\omega_{z}\right)$, the condition

$$
\sum_{K \in \mathcal{T}_{z}} \int_{K} \Phi_{K}^{(3)} u w=0 \quad \forall w \in \mathbb{P}_{p-1}\left(\mathcal{T}_{z}\right)
$$

implies $u=0$.

For a proof we refer to [13, Theorem 1.1]. A consequence of Theorem 5.3 is the following corollary. To reduce technicalities we make an assumption on the minimal local polynomial degree.

Assumption 5.4 For all $\ell$ and $z \in \mathcal{N}_{\ell}^{1}$ it holds: If all $K \subset \omega_{z}$ will be p-refined then $p_{z} \geq 2$ otherwise $p_{z} \geq 1$.

Corollary 5.5 Let Assumption 5.4 be valid. The projection $\Pi_{z}$ is injective.

Proof. If all triangles in $\omega_{z}$ are $p$-refined, then the injectivity follows from Theorem 5.3. If, at least, one triangle is $h$-refined we distinguish between two cases:

a. $K$ is non-regularly $h$-refined. Then, the positivity of $\Phi_{K}^{(1)}$ implies $\left.\left(\Pi_{z} v\right)\right|_{K}=0 \Longrightarrow v=$ 0 .

b. $K$ is regularly $h$-refined. We use the notation as introduced in Figure 3 . Note that $\left.\left(\Pi_{z} v\right)\right|_{K_{1}^{\prime} \cup K_{2}^{\prime}}:=\left.\left(\Phi_{K, K}^{(1)}\left(v+\Pi_{K}^{p_{z}-1} v\right)\right)\right|_{K_{1}^{\prime} \cup K_{2}^{\prime}}$.

b1. If the degree of $v$ satisfies $\operatorname{deg} v=p_{z}-2$, it holds $\Pi_{K}^{p_{z}-1} v=\left.v\right|_{K}$ Then, $\left.\Pi_{z} v\right|_{K_{1}^{\prime} \cup K_{2}^{\prime}}=$ $\left.2 \Phi_{K, K}^{(1)} v\right|_{K_{1}^{\prime} \cup K_{2}^{\prime}}$. The positivity of $\Phi_{K, K}^{(1)}$ on $K_{1}^{\prime} \cup K_{2}^{\prime}$ together with the analytic continuation principle, i.e., $\left.v\right|_{K_{1}^{\prime} \cup K_{2}^{\prime}}=0 \Longrightarrow v=0$, imply the injectivity of $\Pi_{z}$ for this case.

b2. If $\operatorname{deg} v=p_{z}-1$, it holds $v+\Pi_{K}^{p_{z}-1} v \neq 0$. The positivity of $\Phi_{K, K}^{(1)}$ again implies $\left.\left(\Pi_{z} v\right)\right|_{K_{1}^{\prime} \cup K_{2}^{\prime}} \neq 0$.

Corollary 5.6 Let Assumption 5.4 be valid. For all $z \in \mathcal{N}_{\Omega}^{1}$, the estimates

$$
\begin{gathered}
\inf _{v \in \mathbb{P}_{p-1}\left(\omega_{z}\right) \backslash\{0\}} \frac{\left(v, \Pi_{z} v\right)_{L^{2}\left(\omega_{z}\right)}}{\|v\|_{z}^{2}} \geq c_{\pi} . \\
c\left\|\Phi_{z}^{-1 / 2} \Pi_{z} v\right\|_{L^{2}\left(\omega_{z}\right)} \leq\left(v, \Pi_{z} v\right)_{L^{2}\left(\omega_{z}\right)}^{1 / 2} \leq\|v\|_{z} \leq\|v\|_{L^{2}\left(\omega_{z}\right)} .
\end{gathered}
$$

hold. The constant $0<c_{\pi} \leq 1$ only depends, possibly, on the polynomial degree $p$ and the shape regularity of the mesh. 
Proof. For the proof of (5.8), we distinguish between the following cases.

a) If all triangles in $\omega_{z}$ are $p$-refined, estimate (5.8) for some constant $c_{\pi}>0$ follows from the injectivity of $\Pi_{z}$ via the compactness argument in [13, Theorem 6.4] and the equivalence of norms on the finite dimensional space $\mathbb{P}_{p_{z}-1}\left(\omega_{z}\right)$.

b) At least one triangle in $\omega_{z}$ is $h$-refined. Let $K \subset \omega_{z}$.

b1) $K$ is non-regularly $h$-refined. Then, the positivity of $\Phi_{K}^{(1)} \operatorname{implies}\left(v, \Pi_{z} v\right)_{L^{2}(K)}=$ $\left\|\Phi_{K}^{1 / 2} v\right\|_{L^{2}(K)}^{2}>0$ for all $v \in \mathbb{P}_{p_{z}-1}(K) \backslash\{0\}$.

b2) $K$ is regularly $h$-refined. We use the notation as introduced in Figure 3 . Then,

$$
\begin{aligned}
\left(v, \Pi_{z} v\right)_{L^{2}(K)} & =\left(v, \Phi_{K, K}^{(1)} v\right)_{L^{2}(K)}+\left(v, \Phi_{K} \Pi_{K}^{p_{z}-1} v\right)_{L^{2}(K)} \\
& =\left(v, \Phi_{K, K}^{(1)} v\right)_{L^{2}(K)}+\left(\Pi_{K}^{p_{z}-1} v, \Phi_{K} \Pi_{K}^{p_{z}-1} v\right)_{L^{2}(K)} \\
& =\left\|\sqrt{\Phi_{K, K}^{(1)} v}\right\|_{L^{2}(K)}^{2}+\left\|\sqrt{\Phi_{K}} \Pi_{K}^{p_{z}-1} v\right\|_{L^{2}(K)}^{2} \geq\left\|\sqrt{\Phi_{K, K}^{(1)}} v\right\|_{L^{2}(K)}^{2}
\end{aligned}
$$

Again, the positivity of $\Phi_{K, K}^{(1)}$ on $K_{1}^{\prime} \cup K_{2}^{\prime}$ implies $\left(v, \Pi_{z} v\right)_{L^{2}(K)}>0$ for all $v \in \mathbb{P}_{p_{z}-1}(K) \backslash\{0\}$.

For the estimate (5.9) we again consider the different refinement options separately. It is easy to check that pointwise on $K$, we have $\Phi_{K} \leq 1$ so that $\Phi_{K}^{2} \leq \Phi_{K}$.

a) $K$ is $p$-refined, i.e., $\Phi_{K}:=\Phi_{K}^{(3)}$. Estimate

$$
\left(v, \Pi_{z} v\right)_{L^{2}(K)}=\int_{K} \Phi_{K} v \Pi_{K}^{p_{z}-1} v \leq\left\|\sqrt{\Phi_{K}} v\right\|_{L^{2}(K)}^{2}
$$

holds since $\Pi_{K}^{p_{z}-1}$ is a projection. On the other hand,

$$
\left(v, \Pi_{z} v\right)_{L^{2}(K)}=\int_{K} \Phi_{K}\left(\Pi_{K}^{p_{z}-1} v\right)^{2}=\left\|\Phi_{K}^{-1 / 2} \Pi_{z} v\right\|_{L^{2}(K)}^{2} .
$$

b) $K$ is non-regularly $h$-refined. Then,

$$
\left(v, \Pi_{z} v\right)_{L^{2}(K)}=\left\|\sqrt{\Phi_{K}} v\right\|_{L^{2}(K)}^{2} .
$$

From (5.10b) we get

$$
\left(v, \Pi_{z} v\right)_{L^{2}(K)}=\left\|\Phi_{K}^{-1 / 2} \Phi_{K} v\right\|_{L^{2}(K)}^{2}=\left\|\Phi_{K}^{-1 / 2} \Pi_{z} v\right\|_{L^{2}(K)}^{2} .
$$

c) $K$ is regularly $h$-refined. Then,

$$
\begin{aligned}
\left(v, \Pi_{z} v\right)_{L^{2}(K)} & =\left\|\sqrt{\Phi_{K, K}^{(1)}} v\right\|_{L^{2}(K)}^{2}+\left\|\sqrt{\Phi_{K}} \Pi_{K}^{p_{z}-1} v\right\|_{L^{2}(K)}^{2} \leq\left\|\sqrt{\Phi_{K, K}^{(1)} v}\right\|_{L^{2}(K)}^{2}+\left\|\sqrt{\Phi_{K}} v\right\|_{L^{2}(K)}^{2} \\
& \leq 2\left\|\sqrt{\Phi_{K}} v\right\|_{L^{2}(K)}^{2} .
\end{aligned}
$$


For the first estimate in (5.9) we use the pointwise estimate on $K$

$$
\begin{aligned}
\left(\Pi_{z} v\right)^{2} & =\left(\Phi_{K, K}^{(1)} v+\Phi_{K} \Pi_{K}^{p_{z}-1} v\right)^{2} \\
& \leq 2\left(\left(\Phi_{K, K}^{(1)} v\right)^{2}+\left(\Phi_{K} \Pi_{K}^{p_{z}-1} v\right)^{2}\right) \\
& \leq 2\left(\Phi_{K, K}^{(1)}+\Phi_{K}\right)\left(\Phi_{K, K}^{(1)} v^{2}+\Phi_{K}\left(\Pi_{K}^{p_{z}-1} v\right)^{2}\right) \\
& \leq 4 \Phi_{K}\left(\Phi_{K, K}^{(1)} v^{2}+\Phi_{K}\left(\Pi_{K}^{p_{z}-1} v\right)^{2}\right)
\end{aligned}
$$

to get

$$
\int_{K} \Phi_{K}^{-1}\left(\Pi_{z} v\right)^{2} \leq 4\left(\left\|\sqrt{\Phi_{K, K}^{(1)} v}\right\|_{L^{2}(K)}^{2}+\left\|\sqrt{\Phi_{K}} \Pi_{K}^{p_{z}-1} v\right\|_{L^{2}(K)}^{2}\right)=4\left(v, \Pi_{z} v\right)_{L^{2}(K)} .
$$

The second estimate in (5.9) follows by summing the inequality (5.11c) over all $K \subset \omega_{z}$ while the third one is a consequence of $\|v\|_{z} \leq\|v\|_{L^{2}\left(\omega_{z}\right)}$ since $0 \leq \Phi_{z} \leq 1$.

The first estimate in (5.9) also follows by summation over all $K \subset \omega_{z}$ the inequalities (5.11).

The derivation of a sharp positive lower bound for $c_{\pi}$ seems to rather involved. Instead we have performed numerical experiments (cf. Appendix A) to support the following conjecture.

Conjecture 5.7 The constant $c_{\pi}$ is bounded from below by a constant $c_{0}>0$ which only depends on the shape regularity of the mesh but neither on the mesh width nor on the polynomial degree $p$.

For $z \in \mathcal{N}_{\ell}^{1}$, we introduce the subspaces for $K \in \mathcal{T}_{\ell}$ (recall Notation 3.4)

$$
S_{\ell+1, K}:=\left\{u \in S_{\ell+1} \mid \operatorname{supp} u \subset K\right\} \quad \text { and } \quad S_{\ell+1, z}:=\left\{u \in S_{\ell+1} \mid \operatorname{supp} u \subset \omega_{z}\right\} .
$$

Theorem 5.8 Let Assumption 3.1 and 5.4 be satisfied. For $z \in \mathcal{N}_{\ell}^{1}, E \in \mathcal{E}_{z}$, and $w \in S_{\ell}$, let $J_{E}(w):=\left\langle\mathbf{A n}_{E},[\nabla w]_{E}\right\rangle$. Set $p:=p_{z}\left(c f\right.$. (4.5)). For any $v \in \mathbb{P}_{p-1}\left(\omega_{z}\right)$, there exists a $\varphi_{\ell+1, z} \in S_{\ell+1, z}$ such that

$$
\begin{aligned}
& \sum_{E \in \mathcal{E}_{z}} \int_{E} J_{E} b_{z}^{1}=\sum_{E \in \mathcal{E}_{z}} \int_{E} J_{E} \varphi_{\ell+1, z}, \\
& c_{\pi}\left\|\mathfrak{h}_{\ell} v\right\|_{z} \leq\left|\int_{\omega_{z}} v\left(b_{z}^{1}-\varphi_{\ell+1, z}\right)\right|, \\
& \left\|\Phi_{z}^{-1 / 2}\left(b_{z}^{1}-\varphi_{\ell+1, z}\right)\right\|_{L^{2}\left(\omega_{z}\right)}+c_{2} \frac{h_{z}}{p}\left\|\left(b_{z}^{1}-\varphi_{\ell+1, z}\right)\right\|_{\mathrm{PDE}, \omega_{z}} \leq C_{1} h_{z} .
\end{aligned}
$$

The constant $c_{2}>0$ only depends on $\alpha, \beta, b_{\infty}, c_{\infty}$, and the shape-regularity of the mesh while $C_{1}$ is a number.

Proof. We make the ansatz

$$
\varphi_{\ell+1, z}=b_{z}^{1}-\psi_{\ell+1, z}
$$

for some $\psi_{\ell+1, z}$ with $\left.\psi_{\ell+1, z}\right|_{K} \in S_{\ell+1, K}$ for all $K \subset \omega_{z}$. Hence $\left.\psi_{\ell+1, z}\right|_{K}$ vanishes on all edges and condition (5.13a) trivially is satisfied. 
Statement $(5.13 \mathrm{~b})$ is trivial for $v=0$ and we consider here $v \in \mathbb{P}_{p-1}\left(\omega_{z}\right) \backslash\{0\}$. Let

$$
\psi_{\ell+1, z}=h_{z} \frac{\Pi_{z} v}{\|v\|_{z}}
$$

and observe that $\psi_{\ell+1, z} \in S_{\ell+1, z}$. Hence, by Corollary 5.6 we obtain

$$
\left|\int_{\omega_{z}} v\left(b_{z}^{1}-\varphi_{\ell+1, z}\right)\right|=h_{z} \frac{\left|\left(v, \Pi_{z} v\right)_{L^{2}\left(\omega_{z}\right)}\right|}{\|v\|_{z}} \geq c_{\pi} h_{z}\|v\|_{z} .
$$

Finally, we consider estimate (5.13c) and get

$$
\left\|\Phi_{z}^{-1 / 2}\left(b_{z}^{1}-\varphi_{\ell+1, z}\right)\right\|_{L^{2}\left(\omega_{z}\right)}=h_{z} \frac{\left\|\Phi_{z}^{-1 / 2} \Pi_{z} v\right\|_{L^{2}\left(\omega_{z}\right)}}{\|v\|_{z}} \stackrel{(5.9)}{\leq} \frac{h_{z}}{c} \frac{\left(v, \Pi_{z} v\right)_{L^{2}\left(\omega_{z}\right)}^{1 / 2}}{\|v\|_{z}} \stackrel{(5.9)}{\leq} \tilde{C} h_{z} .
$$

For the $H^{1}$-seminorm we get

$$
\left\|\nabla\left(b_{z}^{1}-\varphi_{\ell+1, z}\right)\right\|_{L^{2}\left(\omega_{z}\right)}=h_{z}\left\|\nabla\left(\frac{\Pi_{z} v}{\|v\|_{z}}\right)\right\|_{L^{2}\left(\omega_{z}\right)} .
$$

We distinguish again three cases.

Case a. Let $K \subset \omega_{z}$ be a triangle which will be $p$-refined. Hence, $\Phi_{K}=\Phi_{K}^{(3)}$ (cf. Definition 5.1).

We apply Lemma B.3 to obtain

$$
\left\|\nabla\left(\Pi_{z} v\right)\right\|_{L^{2}(K)}^{2} \leq C_{0}^{\prime} \frac{p^{2}}{h_{z}^{2}}\left\|\Phi_{K}^{1 / 2} \Pi_{K}^{p_{z}-1} v\right\|_{L^{2}(K)}^{2} \leq C_{0}^{\prime} \frac{p^{2}}{h_{z}^{2}}\left\|\Phi_{K}^{1 / 2} v\right\|_{L^{2}(K)}^{2} .
$$

The last inequality in (5.16a) is trivial for $p_{K}>p$ since $\left.\left(\Pi_{z} v\right)\right|_{K}=v$, while, for $p_{K}=p$, we employ (5.10a) and (5.11a).

Case b. Let $K \subset \omega_{z}$ be a triangle which is non-regularly $h$-refined. Hence, $\Phi_{K}=\Phi_{K}^{(1)}$. We introduce the function ${ }^{5} d_{K}: K \rightarrow \mathbb{R}$ by

$$
d_{K}=d_{\widehat{K}} \circ \Lambda_{K}^{-1} \quad \text { with } \quad d_{\widehat{K}}(x):=\operatorname{dist}(x, \partial \widehat{K})
$$

where $\Lambda_{K}$ is as in (5.3). Since both, $\Phi_{K}^{(1)}$ and $d_{K}$ are piecewise linear bubble functions with maximal value $\mathcal{O}(1)$ in the interior it is easy to verify that the pointwise estimates hold

$$
\left.\begin{array}{c}
c d_{K} \leq \Phi_{K}^{(1)} \leq C d_{K} \\
c\left\|\nabla d_{K}\right\| \leq\left\|\nabla \Phi_{K}^{(1)}\right\| \leq C\left\|\nabla d_{K}\right\|
\end{array}\right\} \quad \text { a.e. }
$$

with fixed constants $0<c, C=O(1)$. Estimates (5.17) imply the pointwise estimate

$$
\frac{1}{2}\left\|\nabla\left(v \Phi_{K}\right)\right\|^{2} \leq \Phi_{K}^{2}\|\nabla v\|^{2}+\left\|\nabla \Phi_{K}\right\|^{2} v^{2} \leq C^{2}\left(d_{K}^{2}\|\nabla v\|^{2}+v^{2}\left\|\nabla d_{K}\right\|^{2}\right) .
$$

\footnotetext{
${ }^{5}$ The function $d_{K}$ differs from the function $\Phi_{K}$ in $[16,(27)]$ only by a scaling constant which is of order 1.
} 
Hence, we may use [16, (23) with $\delta=1$ and (22) with $\alpha=0$ and $\beta=1]$ to obtain $\frac{1}{2}\left\|\nabla\left(\Pi_{z} v\right)\right\|_{L^{2}(K)}^{2}=\frac{1}{2}\left\|\nabla\left(v \Phi_{K}\right)\right\|_{L^{2}(K)}^{2} \leq \frac{C^{2}}{h_{K}^{2}}\left(p^{2}\left\|\Phi_{K}^{1 / 2} v\right\|_{L^{2}(K)}^{2}+\|v\|_{L^{2}(K)}^{2}\right) \leq \tilde{C}^{2} \frac{p^{2}}{h_{K}^{2}}\left\|\Phi_{K}^{1 / 2} v\right\|_{L^{2}(K)}^{2}$.

Case c. Let $K \subset \omega_{z}$ be a triangle which is regularly $h$-refined. We employ the notation as explained in Definition 5.1(b1); illustrated in Figure 3. It holds

$$
\begin{aligned}
\nabla\left(\left.\left(\Pi_{z} v\right)\right|_{K}\right) & =\nabla\left(\Phi_{K, K}^{(1)} v+\Phi_{K} \Pi_{K}^{p_{z}-1} v\right) \\
& =\nabla\left(\Phi_{K, K}^{(1)}\left(v+\Pi_{K}^{p_{z}-1} v\right)\right)+\nabla\left(\Phi_{K}^{(2)} \Pi_{K}^{p_{z}-1} v\right)
\end{aligned}
$$

For the first term and the piecewise linear bubble $\Phi_{K, K}^{(1)}$ we can argue as in Case b to obtain

$$
\left\|\nabla\left(\Phi_{K, K}^{(1)}\left(v+\Pi_{K}^{p_{z}-1} v\right)\right)\right\|_{L^{2}(K)}^{2} \leq \tilde{C}^{2} \frac{p^{2}}{h_{K}^{2}}\left\|\sqrt{\Phi_{K, K}^{(1)}}\left(v+\Pi_{K}^{p_{z}-1} v\right)\right\|_{L^{2}(K)}^{2}
$$

while we employ Lemma B.4 for

$$
\left\|\nabla\left(\Phi_{K}^{(2)} \Pi_{K}^{p_{z}-1} v\right)\right\|_{L^{2}(K)}^{2} \leq \hat{C}^{2} \frac{p^{2}}{h_{K}^{2}}\left\|\sqrt{\Phi_{K, K}^{(1)}}\left(\Pi_{K}^{p_{z}-1} v\right)\right\|_{L^{2}(K)}^{2}
$$

Thus,

$$
\begin{aligned}
& \left\|\nabla\left(\left.\left(\Pi_{z} v\right)\right|_{K}\right)\right\|_{L^{2}(K)}^{2} \leq 2\left(\left\|\nabla\left(\Phi_{K, K}^{(1)}\left(v+\Pi_{K}^{p_{z}-1} v\right)\right)\right\|_{L^{2}(K)}^{2}+\left\|\nabla\left(\Phi_{K}^{(2)} \Pi_{K}^{p_{z}-1} v\right)\right\|_{L^{2}(K)}^{2}\right) \\
& \stackrel{(5.18)}{\leq} 2\left(\tilde{C}^{2}+\hat{C}^{2}\right) \frac{p^{2}}{h_{K}^{2}}\left(\left\|\sqrt{\Phi_{K, K}^{(1)}}\left(v+\Pi_{K}^{p_{z}-1} v\right)\right\|_{L^{2}(K)}^{2}+\left\|\sqrt{\Phi_{K}^{(2)}}\left(\Pi_{K}^{p_{z}-1} v\right)\right\|_{L^{2}(K)}^{2}\right) \\
& \leq 4\left(\tilde{C}^{2}+\hat{C}^{2}\right) \frac{p^{2}}{h_{K}^{2}}\left(\left\|\sqrt{\Phi_{K, K}^{(1)}} v\right\|_{L^{2}(K)}^{2}+\left\|\sqrt{\Phi_{K, K}^{(1)}} \Pi_{K}^{p_{z}-1} v\right\|_{L^{2}(K)}^{2}+\left\|\sqrt{\Phi_{K}^{(2)}}\left(\Pi_{K}^{p_{z}-1} v\right)\right\|_{L^{2}(K)}^{2}\right) \\
& =4\left(\tilde{C}^{2}+\hat{C}^{2}\right) \frac{p^{2}}{h_{K}^{2}}\left(\left\|\sqrt{\Phi_{K, K}^{(1)}} v\right\|_{L^{2}(K)}^{2}+\left\|\sqrt{\Phi_{K}} \Pi_{K}^{p_{z}-1} v\right\|_{L^{2}(K)}^{2}\right) \\
& \stackrel{(5.16 \mathrm{a})}{\leq} 4\left(\tilde{C}^{2}+\hat{C}^{2}\right) \frac{p^{2}}{h_{K}^{2}}\left(\left\|\sqrt{\Phi_{K, K}^{(1)} v}\right\|_{L^{2}(K)}^{2}+\left\|\sqrt{\Phi_{K}} v\right\|_{L^{2}(K)}^{2}\right) \\
& \leq 8\left(\tilde{C}^{2}+\hat{C}^{2}\right) \frac{p^{2}}{h_{K}^{2}}\left\|\sqrt{\Phi_{K}} v\right\|_{L^{2}(K)}^{2}
\end{aligned}
$$

The combination of (5.16) with (5.15) leads to

$$
\left\|\nabla \varphi_{\ell+1, z}\right\|_{L^{2}\left(\omega_{z}\right)} \leq \tilde{C}_{0} p
$$




\section{The Saturation Property}

Note that the Pythagoras theorem

$$
\left\|e_{\ell}\right\|_{\mathrm{PDE}}^{2}=\left\|e_{\ell+1}\right\|_{\mathrm{PDE}}^{2}+\left\|u_{\ell}-u_{\ell+1}\right\|_{\mathrm{PDE}}^{2}
$$

only holds for symmetric bilinear forms, i.e., $\mathbf{b}=\mathbf{0}$ in (2.2). For non-symmetric bilinear forms one can prove a quasi-orthogonality and we follow here [14, Proof of Lemma 2.1.]. One ingredient in the proof is an Aubin-Nitsche argument (see, e.g., [9]) which we recall here. For $0<s \leq 1$, we say that the adjoint problem

$$
\text { For given } g \in L^{2}(\Omega) \quad \text { find } \psi_{g} \in H_{0}^{1}(\Omega) \quad \text { such that } \quad a\left(v, \psi_{g}\right):=\int_{\Omega} g v \quad \forall v \in H_{0}^{1}(\Omega)
$$

is $H^{1+s}(\Omega)$-regular if, for any right-hand side $g \in L^{2}(\Omega)$, the solution $\psi_{g}$ is in $H^{1+s}(\Omega)$ and there exists a constant $C_{s}$ independent of $g \in L^{2}(\Omega)$ such that

$$
\left\|\psi_{g}\right\|_{H^{1+s}(\Omega)} \leq C_{s}\|g\|_{L^{2}(\Omega)}
$$

We introduce the adjoint approximation property for a subspace $S \subset H_{0}^{1}(\Omega)$ by

$$
\eta(S):=\sup _{g \in L^{2}(\Omega) \backslash\{0\}} \inf _{v \in S} \frac{\left\|\psi_{g}-v\right\|_{\mathrm{PDE}}}{\|g\|_{L^{2}(\Omega)}} .
$$

In our context, we obtain, e.g., from [9] the estimate

$$
\left\|e_{\ell+1}\right\|_{L^{2}(\Omega)} \leq C_{S} \eta\left(S_{\ell+1}\right)\left\|e_{\ell+1}\right\|_{\mathrm{PDE}} .
$$

If the adjoint problem is $H^{1+s}(\Omega)$-regular, standard approximation results for finite elements lead to

$\eta\left(S_{\ell}\right) \leq C_{a} \sup _{g \in L^{2}(\Omega) \backslash\{0\}} \inf _{v \in S_{\ell}} \frac{\left\|\psi_{g}-v\right\|_{H^{1}(\Omega)}}{\|g\|_{L^{2}(\Omega)}} \leq C_{a} C_{\text {approx }} h_{\ell}^{s} \sup _{g \in L^{2}(\Omega) \backslash\{0\}} \frac{\left\|\psi_{g}\right\|_{H^{1+s}(\Omega)}}{\|g\|_{L^{2}(\Omega)}} \leq C_{a} C_{\text {approx }} C_{s} h_{\ell}^{s}$,

where $C_{\text {approx }}$ only depends on the shape regularity of the mesh. Hence,

$$
\left\|e_{\ell+1}\right\|_{L^{2}(\Omega)} \leq C_{\text {dual }} h_{\ell+1}^{s}\left\|e_{\ell+1}\right\|_{\mathrm{PDE}} \quad \text { with } \quad C_{\text {dual }}:=C_{S} C_{a} C_{\text {approx }} C_{s} .
$$

Lemma 6.1 Let Assumption 2.1 be satisfied and let the adjoint problem be $H^{1+s}(\Omega)$ regular for some $0<s \leq 1$. Then, there exists some $C_{\star}>0$ depending only on $\alpha, \beta, b_{\infty}, c_{\infty}$, and the shape regularity of the mesh such that, for any finite element mesh $\mathcal{T}_{\ell+1}$ with maximal mesh width $h_{\ell+1}<C_{\star}^{-s}$, the quasi-orthogonality

$$
\left\|e_{\ell+1}\right\|_{\mathrm{PDE}}^{2} \leq \Lambda_{\ell+1}^{2}\left\|e_{\ell}\right\|_{\mathrm{PDE}}^{2}-\left\|u_{\ell+1}-u_{\ell}\right\|_{\mathrm{PDE}}^{2} \quad \text { with } \quad \Lambda_{\ell+1}^{2}:=\frac{1}{1-C_{\star} h_{\ell+1}^{s}}
$$

holds. 
The proof is adapted from [14, Lem. 4.1] and included here for completeness.

Proof. We set $\varepsilon_{\ell}:=u_{\ell+1}-u_{\ell}$. It is easy to conclude from Galerkin's orthogonality that

$$
\left\|e_{\ell}\right\|_{\mathrm{PDE}}^{2}=\left\|e_{\ell+1}\right\|_{\mathrm{PDE}}^{2}+\left\|\varepsilon_{\ell}\right\|_{\mathrm{PDE}}^{2}+a\left(\varepsilon_{\ell}, e_{\ell+1}\right)
$$

holds. Then, integration by parts yields

$$
\begin{aligned}
a\left(\varepsilon_{\ell}, e_{\ell+1}\right) & =a\left(e_{\ell+1}, \varepsilon_{\ell}\right)+\int_{\Omega}\left(\left\langle\mathbf{b}, \nabla \varepsilon_{\ell}\right\rangle e_{\ell+1}-\left\langle\mathbf{b}, \nabla e_{\ell+1}\right\rangle \varepsilon_{\ell}\right) \\
& =\int_{\Omega}\left(2\left\langle\mathbf{b}, \nabla \varepsilon_{\ell}\right\rangle+(\operatorname{div} \mathbf{b}) \varepsilon_{\ell}\right) e_{\ell+1} .
\end{aligned}
$$

Hence,

$$
\left\|e_{\ell+1}\right\|_{\mathrm{PDE}}^{2}=\left\|e_{\ell}\right\|_{\mathrm{PDE}}^{2}-\left\|\varepsilon_{\ell}\right\|_{\mathrm{PDE}}^{2}-\int_{\Omega}\left(2\left\langle\mathbf{b}, \nabla \varepsilon_{\ell}\right\rangle+(\operatorname{div} \mathbf{b}) \varepsilon_{\ell}\right) e_{\ell+1} .
$$

The integral can be estimated by Young's inequality and estimate (6.1)

$$
\begin{aligned}
-\int_{\Omega}\left(2\left\langle\mathbf{b}, \nabla \varepsilon_{\ell}\right\rangle+(\operatorname{div} \mathbf{b}) \varepsilon_{\ell}\right) e_{\ell+1} & \leq \delta\left\|e_{\ell+1}\right\|_{L^{2}(\Omega)}^{2}+\frac{\left(3 b_{\infty}\right)^{2}}{2 \delta}\left\|\varepsilon_{\ell}\right\|_{H^{1}(\Omega)}^{2} \\
& \stackrel{(2.3)}{\leq} \delta\left\|e_{\ell+1}\right\|_{L^{2}(\Omega)}^{2}+\frac{9 b_{\infty}^{2} c_{\mathrm{F}}}{2 \delta \alpha}\left\|\varepsilon_{\ell}\right\|_{\mathrm{PDE}}^{2} \\
& \leq \delta C_{\mathrm{dual}}^{2} h_{\ell+1}^{2 s}\left\|e_{\ell+1}\right\|_{\mathrm{PDE}}^{2}+\frac{9 b_{\infty}^{2} c_{\mathrm{F}}}{2 \delta \alpha}\left\|\varepsilon_{\ell}\right\|_{\mathrm{PDE}}^{2} .
\end{aligned}
$$

Inserting this into (6.3) leads to

$$
\left(1-\delta C_{\text {dual }}^{2} h_{\ell+1}^{2 s}\right)\left\|e_{\ell+1}\right\|_{\mathrm{PDE}}^{2} \leq\left\|e_{\ell}\right\|_{\mathrm{PDE}}^{2}-\left(1-\frac{9 b_{\infty}^{2} c_{\mathrm{F}}}{2 \delta \alpha}\right)\left\|\varepsilon_{\ell}\right\|_{\mathrm{PDE}}^{2} .
$$

We choose $\delta$ such that both parenthesis have the same value and obtain

$$
\left\|e_{\ell+1}\right\|_{\mathrm{PDE}}^{2} \leq \frac{\left\|e_{\ell}\right\|_{\mathrm{PDE}}^{2}}{1-C_{\star} h_{\ell+1}^{s}}-\left\|\varepsilon_{\ell}\right\|_{\mathrm{PDE}}^{2} \quad \text { with } \quad C_{\star}:=3 C_{\mathrm{dual}} b_{\infty} \sqrt{\frac{c_{\mathrm{F}}}{2 \alpha}} .
$$

Let the mesh width $h_{\ell+1}$ of $\mathcal{T}_{\ell+1}$ satisfy $h_{\ell+1}^{s}<C_{\star}^{-1}$. Then the assertion holds with $\Lambda_{\ell+1}^{2}$ as in (6.2).

The proof of the saturation estimate requires conditions on the data oscillations. First, we will introduce some edge bubble for triangles with a common edge. For $E \in \mathcal{E}_{\Omega}$, let $K_{1}, K_{2} \in \mathcal{T}_{\ell}$ denote the triangles which share $E$ as the common edge.

Case a) Both, $K_{1}, K_{2}$ will be $p$-refined.

In this case, let $\varphi_{K_{1}, K_{2}}^{(2)} \in S_{\ell+1}$ be the quadratic edge bubble, i.e., the product of the barycentric coordinates in $K_{1}, K_{2}$ for the endpoints of $E$.

Case b) Both $K_{1}, K_{2}$ will be $h$-refined

Let $x_{E} \in E$ denote the interior vertex on the edge $E$ (cf. Figure 1) and let $K \in\left\{K_{1}, K_{2}\right\}$ be an adjacent triangle with inner vertex $x_{K}$. Let $K^{\prime}:=\operatorname{conv}\left\{x_{E}, x_{K}, A\right\}$ and $K^{\prime \prime}:=$ conv $\left\{x_{E}, B, x_{K}\right\}$ with $A, B$ denoting the endpoints of $E$. Then, the piecewise affine edge bubble $\varphi_{K_{1}, K_{2}}^{(1)}$, restricted to $K$, has value 1 at $x_{E}$ and vanishes at all other vertices of triangles in sons $\left(K_{1}\right)$. Assumption 3.1 ensures that $\varphi_{K_{1}, K_{2}}^{(1)} \in S_{\ell+1}$. 
Case c) $K_{1}$ will be $p$-refined and $K_{2}$ will be $h$-refined. Let $p_{m}:=p_{K_{m}}, m=1,2$ and define $p_{E}:=\min \left\{p_{1}, p_{2}\right\}$. Let $K^{\prime} \in \operatorname{sons}\left(K_{2}\right)$ be the triangle which contains $E$ as an edge. Then, $\left.\varphi_{K_{1}, K^{\prime}}^{(2)}\right|_{K_{1}}$ (resp. $\left.\varphi_{K_{1}, K^{\prime}}^{(2)}\right|_{K^{\prime}}$ ) is the product of the barycentric coordinates in $K_{1}$ (resp. $K^{\prime}$ ) for the endpoints of $E$ and zero outside $\overline{K_{1} \cup K^{\prime}}$.

Case d) $K_{1}$ will be $h$-refined and $K_{2}$ will be $p$-refined. Then $\varphi_{K^{\prime}, K_{2}}^{(2)}$ is defined as in Case c by interchanging the roles of $K_{1}$ and $K_{2}$.

We define

$$
\varphi_{E}:= \begin{cases}\varphi_{K_{1}, K_{2}}^{(2)} & \text { in Case a } \\ \varphi_{K_{1}, K_{2}}^{(1)} & \text { in Case b } \\ \varphi_{K_{1}, K^{\prime}}^{(2)} & \text { in Case c } \\ \varphi_{K^{\prime}, K_{2}}^{(2)} & \text { in Case d }\end{cases}
$$

For $g \in L^{2}(\Omega)$, we define averages $g_{z} \in \mathbb{P}_{p_{z}-1}\left(\omega_{z}\right)$ (with $p_{z}$ as in (4.5)) as the $L^{2}\left(\omega_{z}\right)$ orthogonal projection onto $\mathbb{P}_{p_{z}-1}\left(\omega_{z}\right)$.

The data oscillations are defined by

$$
\operatorname{osc}(v):=\sqrt{\sum_{z \in \mathcal{N}_{\ell}^{1}} \operatorname{osc}_{z}^{2}(v)} \quad \text { with } \quad \operatorname{osc}_{z}(v):=\left\|\frac{h_{z}}{p_{z}} \Phi_{\text {osc }, z}^{1 / 2}\left(\operatorname{res}(v)-\operatorname{res}_{z}(v)\right)\right\|_{L^{2}\left(\omega_{z}\right)}
$$

with

$$
\Phi_{\mathrm{osc}, z}:=\frac{p_{z}^{5}}{c_{\pi}^{2}} \Phi_{z}+p_{z}^{3} \Phi_{\mathcal{E}, z}+1 \quad \text { and } \quad \Phi_{\mathcal{E}, z}:=\sum_{E \in \mathcal{E}_{z}} \varphi_{E} .
$$

and $\operatorname{res}_{z}(v)$ is a shorthand for $(\operatorname{res}(v))_{z}$.

Theorem 6.2 Let Assumptions 2.1, 3.1, and 5.4 be satisfied. We assume that the adjoint problem is $H^{1+s}(\Omega)$ regular for some $0<s \leq 1$. Further we assume that the maximal mesh width of $\mathcal{T}_{\ell+1}$ satisfies $h_{\ell+1}<C_{\star}^{-s}$ with $C_{\star}$ as in Lemma 6.1. Let $c_{\pi}$ be as in (5.13b) and $C_{\text {rel }}$ as in (4.1).

There exists a constant $C_{2}>0$ depending on $\alpha, \beta, b_{\infty}, c_{\infty}$, and $\rho$ but independent of $\mathfrak{h}_{\ell}, \mathbf{p}_{\ell}$, $u$, and $f$ such that for any $0 \leq \mu \leq 1$ and any $C_{3}>C_{2} C_{\text {rel }}$ the condition

$$
\operatorname{osc}\left(u_{\ell}\right) \leq \frac{\mu}{C_{3}}\left\|e_{\ell}\right\|_{\mathrm{PDE}}
$$

implies the error reduction

$$
\left\|e_{\ell+1}\right\|_{\mathrm{PDE}} \leq \kappa_{\ell}\left\|e_{\ell}\right\|_{\mathrm{PDE}} \quad \text { with } \quad \kappa_{\ell}:=\sqrt{\left(\Lambda_{\ell+1}^{2}-\left(\frac{c_{\pi}}{C_{3} p_{\ell}^{5 / 2}}\right)^{2}\left(1-\mu^{2}\right)\right)}
$$

Remark 6.3 The condition on $C_{3}$ implies that $\kappa_{\ell}>0$. From the definition of $\Lambda_{\ell+1}^{2}=\frac{1}{1-C_{\star} h_{\ell+1}^{s}}$ with $h_{\ell+1}<\left(2 C_{\star}\right)^{-1 / s}$ as in (6.2) it follows that the condition

$$
h_{\ell+1} \leq H\left(p_{\ell}\right):=C_{4}\left(\frac{c_{\pi}}{p_{\ell}^{5 / 2}}\right)^{2 / s} \quad \text { with } \quad C_{4}:=\left(\frac{1}{C_{\star}\left(2 C_{3} C_{\mathrm{rel}}\right)^{2}}\right)^{1 / s}
$$


implies

$$
\Lambda_{\ell+1}^{2}-\left(\frac{c_{\pi}}{C_{3} C_{\mathrm{rel}} p_{\ell}^{5 / 2}}\right)^{2} \leq 1-\varepsilon_{\ell} \quad \text { with } \quad \varepsilon_{\ell}:=\frac{1}{2}\left(\frac{c_{\pi}}{C_{3} C_{\mathrm{rel}} p_{\ell}^{5 / 2}}\right)^{2}
$$

and, for $0 \leq \mu<1 / \sqrt{2}$, it holds

$$
\kappa_{\ell} \leq \sqrt{1-\left(\frac{c_{\pi}}{C_{3} C_{\mathrm{rel}} p_{\ell}^{5 / 2}}\right)^{2}\left(\frac{1}{2}-\mu^{2}\right)}<1
$$

Proof of Theorem 6.2. Since $u_{\ell+1}-u_{\ell} \in S_{\ell+1}$, the quasi-orthogonality (cf. Lemma 6.1) implies

$$
\Lambda_{\ell+1}^{2}\left\|e_{\ell}\right\|_{\mathrm{PDE}}^{2} \geq\left\|e_{\ell+1}\right\|_{\mathrm{PDE}}^{2}+\left\|u_{\ell+1}-u_{\ell}\right\|_{\mathrm{PDE}}^{2}
$$

with $\Lambda_{\ell+1}^{2}$ as in (6.2). Hence it is sufficient to prove a lower bound for $\left\|u_{\ell+1}-u_{\ell}\right\|_{\mathrm{PDE}}^{2}$ in terms of $\left\|e_{\ell}\right\|_{\mathrm{PDE}}^{2}$ and data oscillations. The residual a posteriori error estimate can be recast in the form of stars (cf. (4.4)): By a triangle inequality we obtain

$$
\begin{aligned}
\left\|e_{\ell}\right\|_{\mathrm{PDE}}^{2} & \leq 2 C_{\mathrm{rel}}^{2} \sum_{z \in \mathcal{N}_{\ell}^{1}}\left(\left\|\frac{h_{z}}{p_{z}} \operatorname{res}_{z}\left(u_{\ell}\right)\right\|_{L^{2}\left(\omega_{z}\right)}^{2}+\sum_{E \in \mathcal{E}_{z}}\left\|\sqrt{\frac{h_{z}}{p_{z}}} \operatorname{Res}\left(u_{\ell}\right)\right\|_{L^{2}(E)}^{2}\right. \\
& \left.+\left\|\frac{h_{z}}{p_{z}}\left(\operatorname{res}_{z}\left(u_{\ell}\right)-\operatorname{res}\left(u_{\ell}\right)\right)\right\|_{L^{2}\left(\omega_{z}\right)}^{2}\right)
\end{aligned}
$$

Hence, it is sufficient to bound the jumps and projected volume residuals from above by $\left\|u_{\ell+1}-u_{\ell}\right\|_{\mathrm{PDE}}$ and to control the last term by the oscillation condition (6.6).

We start with the jump term and employ the same arguments as in [14, Proof of Lemma 3.1, Step 2]. Since $u_{\ell}$ is continuous, $\left[\nabla u_{\ell}\right]_{E}$ is parallel to $\mathbf{n}_{E}$, i.e., $\left[\nabla u_{\ell}\right]_{E}=j_{E} \mathbf{n}_{E}$ and $j_{E}=$ $\left[\frac{\partial u_{\ell}}{\partial \mathbf{n}_{E}}\right]_{E} \in \mathbb{P}_{p_{E}-1}$ with $p_{E}:=\min \left\{p_{1}, p_{2}\right\}$ (cf. Remark 3.3). The continuity of the coefficient matrix A implies

$$
J_{E}:=\left\langle\mathbf{A} \mathbf{n}_{E},\left[\nabla u_{\ell}\right]_{E}\right\rangle=\left\langle\mathbf{A} \mathbf{n}_{E}, \mathbf{n}_{E}\right\rangle j_{E}=: a_{E} j_{E},
$$

where $\alpha \leq a_{E}(x) \leq \beta$ (cf. Assumption 2.1). Consequently

$$
\begin{aligned}
& \left\|\sqrt{\varphi_{E}} J_{E}\right\|_{L^{2}(E)}^{2}=\int_{E} \alpha_{E}\left(j_{E} \varphi_{E}\right) J_{E} \leq \beta \int_{E}\left(j_{E} \varphi_{E}\right) J_{E} \\
& \left\|\sqrt{\varphi_{E}} J_{E}\right\|_{L^{2}(E)}^{2}=\int_{E} \alpha_{E}^{2} j_{E}^{2} \varphi_{E} \geq \alpha^{2} \int_{E} j_{E}^{2} \varphi_{E} \stackrel{[16, \text { Lem. 2.4] }}{\geq} c \frac{\alpha^{2}}{p_{E}^{2}}\left\|j_{E}\right\|_{L^{2}(E)}^{2} \geq c\left(\frac{\alpha}{\beta p_{E}}\right)^{2}\left\|J_{E}\right\|_{L^{2}(E)}^{2}
\end{aligned}
$$

Thus

$$
\left\|\sqrt{h_{E} \varphi_{E}} J_{E}\right\|_{L^{2}(E)}^{2} \leq \beta h_{E} \int_{E}\left\langle\mathbf{A n},\left[\nabla u_{\ell}\right]_{E}\right\rangle\left(j_{E} \varphi_{E}\right)
$$

Next we extend $j_{E}$ to $\omega_{E}$. For $K \subset \omega_{E}$, let $\Lambda_{K}, \Lambda_{E}$ be chosen such that (cf. (5.3)) $\left.\Lambda_{K}\right|_{x_{2}=0}=$ $\Lambda_{E}$ holds. Let $\hat{Z}(x):=\left(x_{1}, 0\right)^{\top}$ for $x=\left(x_{1}, x_{2}\right) \in \mathbb{R}^{2}$. We define $j_{E}^{\star}: \omega_{E} \rightarrow \mathbb{R}$ trianglewise by

$$
\left.j_{E}^{\star}\right|_{K}:=j_{E} \circ \Lambda_{K} \circ \hat{Z} \circ \Lambda_{K}^{-1}
$$


Note that $j_{E}^{\star}$ is a polynomial of degree $p_{E}-1$ on both triangles which share $E$ as the common edge. The construction of $\varphi_{E}$ along the definition of the polynomial degrees on the refined mesh (cf. Assumption 3.1) imply $\varphi_{E} j_{E}^{\star} \in S_{\ell+1}$. By using partial integration and the fact that $u_{\ell+1}$ is the Galerkin solution we get for any $E \subset \omega_{z}$

$$
\begin{aligned}
\frac{1}{\beta} \| & \sqrt{h_{E} \varphi_{E}} J_{E} \|_{L^{2}(E)}^{2} \leq h_{E} \int_{E}\left\langle\mathbf{A n},\left[\nabla u_{\ell}\right]_{E}\right\rangle\left(j_{E} \varphi_{E}\right) \\
& =h_{E} \int_{\omega_{E}}\left\langle\mathbf{A} \nabla u_{\ell}, \nabla\left(j_{E}^{\star} \varphi_{E}\right)\right\rangle+\operatorname{div}_{\ell}\left(\mathbf{A} \nabla_{\ell} u_{\ell}\right)\left(j_{E}^{\star} \varphi_{E}\right) \\
& =h_{E}\left\{a\left(u_{\ell}-u_{\ell+1}, j_{E}^{\star} \varphi_{E}\right)+\int_{\omega_{E}} \operatorname{res}_{z}\left(u_{\ell}\right)\left(j_{E}^{\star} \varphi_{E}\right)+\left(\operatorname{res}\left(u_{\ell}\right)-\operatorname{res}_{z}\left(u_{\ell}\right)\right)\left(j_{E}^{\star} \varphi_{E}\right)\right\} .
\end{aligned}
$$

From Lemma B.5 and Corollary B.6 we conclude by an affine pullback to the reference element that

$$
\begin{aligned}
\left\|\sqrt{\varphi_{E}} j_{E}^{\star}\right\|_{L^{2}\left(\omega_{E}\right)} & \leq C_{5}\left\|\sqrt{h_{E} \varphi_{E}} j_{E}\right\|_{L^{2}(E)} \stackrel{(6.9)}{\leq} \frac{C_{5}}{\alpha}\left\|\sqrt{h_{E} \varphi_{E}} J_{E}\right\|_{L^{2}(E)}, \\
\left\|\varphi_{E} j_{E}^{\star}\right\|_{\mathrm{PDE}} & \leq C_{6} p_{E}\left\|\sqrt{\frac{\varphi_{E}}{h_{E}}} J_{E}\right\|_{L^{2}(E)},
\end{aligned}
$$

where $C_{5}, C_{6}$ only depend on $\alpha, \beta, b_{\infty}, c_{\infty}$ and the shape regularity of the mesh. The combination of (6.12) with (6.13) and (6.10) leads to

$$
\begin{aligned}
\left\|\sqrt{\frac{h_{E}}{p_{E}}} \operatorname{Res}\left(u_{\ell}\right)\right\|_{L^{2}(E)} & \leq C_{7} p_{E}^{3 / 2}\left\{\left\|u_{\ell}-u_{\ell+1}\right\|_{\mathrm{PDE}, \omega_{E}}+\left\|\frac{h_{E}}{p_{E}} \varphi_{E}^{1 / 2} \operatorname{res}_{z}\left(u_{\ell}\right)\right\|_{L^{2}\left(\omega_{E}\right)}\right. \\
& \left.+\left\|\frac{h_{E}}{p_{E}} \varphi_{E}^{1 / 2}\left(\operatorname{res}\left(u_{\ell}\right)-\operatorname{res}_{z}\left(u_{\ell}\right)\right)\right\|_{L^{2}\left(\omega_{E}\right)}\right\}
\end{aligned}
$$

with $C_{7}$ depending only on $\alpha, \beta, b_{\infty}, c_{\infty}$, and $\rho$. A summation of the squared inequality (6.14) over all $E \in \mathcal{E}_{z}$ yields

$$
\begin{aligned}
\sum_{E \in \mathcal{E}_{z}}\left\|\sqrt{\frac{h_{E}}{p_{E}}} \operatorname{Res}\left(u_{\ell}\right)\right\|_{L^{2}(E)}^{2} & \leq C_{8} p_{z}^{3}\left\{\left\|u_{\ell}-u_{\ell+1}\right\|_{\mathrm{PDE}, \omega_{z}}^{2}+\left\|\frac{h_{z}}{p_{z}} \Phi_{\mathcal{E}, z}^{1 / 2} \operatorname{res}_{z}\left(u_{\ell}\right)\right\|_{L^{2}\left(\omega_{z}\right)}^{2}+\right. \\
& \left.\left\|\frac{h_{z}}{p_{z}} \Phi_{\mathcal{E}, z}^{1 / 2}\left(\operatorname{res}\left(u_{\ell}\right)-\operatorname{res}_{z}\left(u_{\ell}\right)\right)\right\|_{L^{2}\left(\omega_{z}\right)}^{2}\right\} .
\end{aligned}
$$

Hence, we are left with the estimate of the volume residual.

Partial integration and the fact that $u_{\ell}$ solves the Galerkin equations leads to

$$
\begin{aligned}
\sum_{E \in \mathcal{E}_{z}} \int_{E} J_{E} b_{z}^{1} & =\int_{\omega_{z}}\left\langle\mathbf{A} \nabla u_{\ell}, \nabla b_{z}^{1}\right\rangle+\operatorname{div}_{\ell}\left(\mathbf{A} \nabla_{\ell} u_{\ell}\right) b_{z}^{1}=\int_{\omega_{z}} \operatorname{res}\left(u_{\ell}\right) b_{z}^{1} \\
& =\int_{\omega_{z}} \operatorname{res}_{z}\left(u_{\ell}\right) b_{z}^{1}+\int_{\omega_{z}}\left(\operatorname{res}\left(u_{\ell}\right)-\operatorname{res}_{z}\left(u_{\ell}\right)\right) b_{z}^{1} .
\end{aligned}
$$


We choose $\varphi_{\ell+1, z}$ as in Theorem 5.8 such that (5.13) holds and obtain as in (6.12c)

$$
\begin{aligned}
\sum_{E \in \mathcal{E}_{z}} \int_{E} J_{E} \varphi_{\ell+1, z} & =\sum_{E \in \mathcal{E}_{z}} \int_{E}\left\langle\mathbf{A n},\left[\nabla u_{\ell}\right]_{E}\right\rangle \varphi_{\ell+1, z}=\sum_{K \subset \omega_{z}} \int_{K}\left\langle\mathbf{A} \nabla u_{\ell}, \nabla \varphi_{\ell+1, z}\right\rangle+\operatorname{div}_{\ell}\left(\mathbf{A} \nabla u_{\ell}\right) \varphi_{\ell+1, z} \\
& =a\left(u_{\ell}-u_{\ell+1}, \varphi_{\ell+1, z}\right)+\int_{\omega_{z}} \operatorname{res}_{z}\left(u_{\ell}\right) \varphi_{\ell+1, z}+\int_{\omega_{z}}\left(\operatorname{res}\left(u_{\ell}\right)-\operatorname{res}_{z}\left(u_{\ell}\right)\right) \varphi_{\ell+1, z} .
\end{aligned}
$$

The combination of (6.16) and (6.17) with (5.13a) allows to eliminate the jump residuals and we obtain

$$
\int_{\omega_{z}} \operatorname{res}_{z}\left(u_{\ell}\right)\left(b_{z}^{1}-\varphi_{\ell+1, z}\right)=a\left(u_{\ell}-u_{\ell+1}, \varphi_{\ell+1, z}-b_{z}^{1}\right)+\int_{\omega_{z}}\left(\operatorname{res}\left(u_{\ell}\right)-\operatorname{res}_{z}\left(u_{\ell}\right)\right)\left(\varphi_{\ell+1, z}-b_{z}^{1}\right) .
$$

Recall the definition of $\varphi_{\ell+1, z}$ as in the proof of Theorem 5.8

$$
b_{z}^{1}-\varphi_{\ell+1, z}=v_{z} \quad \text { with } \quad v_{z}:=h_{z} \frac{\Pi_{z} v}{\|v\|_{z}}
$$

and we apply this definition for $v=\operatorname{res}_{z}\left(u_{\ell}\right)$. From this and $(5.13 \mathrm{~b})$ we obtain a bound of the averaged volume residual on stars

$$
\begin{aligned}
c_{\pi}\left\|\Phi_{z}^{1 / 2} h_{z} \operatorname{res}_{z}\left(u_{\ell}\right)\right\|_{L^{2}\left(\omega_{z}\right)} & \leq C_{S}\left\|u_{\ell}-u_{\ell+1}\right\|_{\mathrm{PDE}, \omega_{z}}\left\|\varphi_{\ell+1, z}-b_{z}^{1}\right\|_{\mathrm{PDE}, \omega_{z}} \\
& +\left\|h_{z} \Phi_{z}^{1 / 2}\left(\operatorname{res}\left(u_{\ell}\right)-\operatorname{res}_{z}\left(u_{\ell}\right)\right)\right\|_{L^{2}\left(\omega_{z}\right)} h_{z}^{-1}\left(\left\|\Phi_{z}^{-1 / 2}\left(\varphi_{\ell+1, z}-b_{z}^{1}\right)\right\|_{L^{2}\left(\omega_{z}\right)}\right) .
\end{aligned}
$$

Hence, from (5.13) we conclude

$c_{\pi}\left\|\Phi_{z}^{1 / 2} h_{z} \operatorname{res}_{z}\left(u_{\ell}\right)\right\|_{L^{2}\left(\omega_{z}\right)} \leq C_{S} \frac{C_{1}}{c_{2}} p_{z}\left\|u_{\ell}-u_{\ell+1}\right\|_{\mathrm{PDE}, \omega_{z}}+2 C_{1}\left\|h_{z} \Phi_{z}^{1 / 2}\left(\operatorname{res}\left(u_{\ell}\right)-\operatorname{res}_{z}\left(u_{\ell}\right)\right)\right\|_{L^{2}\left(\omega_{z}\right)}$.

Note that ${ }^{6}$

$$
\left\|\Phi_{z}^{1 / 2} h_{z} \operatorname{res}_{z}\left(u_{\ell}\right)\right\|_{L^{2}\left(\omega_{z}\right)} \geq \frac{c}{p_{z}^{1-s}}\left\|\frac{h_{z}}{p_{z}} \Phi_{\mathcal{E}, z}^{s / 2} \operatorname{res}_{z}\left(u_{\ell}\right)\right\|_{L^{2}\left(\omega_{z}\right)} \quad \text { for } s \in\{0,1\} .
$$

\footnotetext{
${ }^{6}$ For $s=1$ this follows from Corollary B.7. For $s=0$, we conclude from [23, Prop. 3.37, Cor. 3.40, Prop. 3.46] that

$$
\left\|\sqrt{\Phi_{K}^{(3)}} v\right\|_{L^{2}(K)} \geq \frac{c}{p^{2}}\|v\|_{L^{2}(K)} \quad \forall v \in \mathbb{P}_{p}(K), \quad p \geq 1
$$
}

holds and from $[16,(22)$ with $\alpha=0$ and $\beta=1]$

$$
\left\|\sqrt{\Phi_{K}^{(1)}} v\right\|_{L^{2}(K)} \geq \frac{c}{p}\|v\|_{L^{2}(K)} \quad \forall v \in \mathbb{P}_{p}(K), \quad p \geq 1
$$

for some constant $c>0$ which is independent of $p$ and $h_{K}$. Finally, for $\Phi_{K, K}^{(1)}+\Phi_{K}^{(2)}$ we employ

$$
\Phi_{K}^{(3)} \leq \Phi_{K, K}^{(1)}+\Phi_{K}^{(2)} \quad \text { pointwise }
$$

to obtain

$$
\left\|\sqrt{\Phi_{K, K}^{(1)}+\Phi_{K}^{(2)}} v\right\|_{L^{2}(K)} \geq\left\|\sqrt{\Phi_{K}^{(3)}} v\right\|_{L^{2}(K)} \geq \frac{c}{p^{2}}\|v\|_{L^{2}(K)} \quad \forall v \in \mathbb{P}_{p}(K), \quad p \geq 1 .
$$


The first and second term in the right-hand side in (6.8) can be estimated by means of (6.14) and (6.20)

$$
\left\|e_{\ell}\right\|_{\mathrm{PDE}}^{2} \leq 2 C_{\mathrm{rel}}^{2} C \sum_{z \in \mathcal{N}_{\ell}^{1}}\left(\frac{p_{z}^{5}}{c_{\pi}^{2}}\left\|u_{\ell}-u_{\ell+1}\right\|_{\mathrm{PDE}, \omega_{z}}^{2}+\left\|\frac{h_{z}}{p_{z}} \Phi_{\mathrm{osc}, z}^{1 / 2}\left(\operatorname{res}\left(u_{\ell}\right)-\operatorname{res}_{z}\left(u_{\ell}\right)\right)\right\|_{L^{2}\left(\omega_{z}\right)}^{2}\right)
$$

where, again, $C_{9}$ only depends on $\alpha, \beta, b_{\infty}, c_{\infty}$, and $\rho$.

Taking into account the finite overlap of the supports $\omega_{z}$ we end up with

$$
\left\|e_{\ell}\right\|_{\mathrm{PDE}}^{2} \leq\left(C_{2} C_{\mathrm{rel}}\right)^{2}\left(\frac{p_{\ell}^{5}}{c_{\pi}^{2}}\left\|u_{\ell}-u_{\ell+1}\right\|_{\mathrm{PDE}}^{2}+\operatorname{osc}^{2}\left(u_{\ell}\right)\right),
$$

where $C_{2}$ only depends on $\alpha, \beta, b_{\infty}, c_{\infty}$, and $\rho$.

Choose $C_{3}>C_{2} C_{\text {rel }}$. The assumption that the data oscillations are small, i.e., osc $\left(u_{\ell}\right) \leq$ $\frac{\mu}{C_{3}}$, implies for any $0 \leq \mu<1$

$$
\sum_{z \in \mathcal{N}_{\ell}^{1}}\left\|u_{\ell}-u_{\ell+1}\right\|_{\mathrm{PDE}, \omega_{z}}^{2} \geq \frac{c_{\pi}^{2}}{C_{3}^{2} p_{\ell}^{5}}\left(1-\mu^{2}\right)\left\|e_{\ell}\right\|_{\mathrm{PDE}}^{2}
$$

The combination with (6.7) finally leads to

$$
\left\|e_{\ell+1}\right\|_{\mathrm{PDE}}^{2} \leq \Lambda_{\ell+1}^{2}\left\|e_{\ell}\right\|_{\mathrm{PDE}}^{2}-\left\|u_{\ell+1}-u_{\ell}\right\|_{\mathrm{PDE}}^{2} \leq\left(\Lambda_{\ell+1}^{2}-\left(\frac{c_{\pi}}{C_{3} p_{\ell}^{5 / 2}}\right)^{2}\left(1-\mu^{2}\right)\right)\left\|e_{\ell}\right\|_{\mathrm{PDE}}^{2}
$$

and this is the assertion.

Corollary 6.4 Let the assumptions of Theorem 6.2 be satisfied. Condition (6.6) follows from the computable condition

$$
\operatorname{osc}\left(u_{\ell}\right) \leq \hat{\mu} \frac{c_{\pi}}{C_{10} p_{\ell}^{5 / 2}}\left(\sum_{E \in \mathcal{E}_{\Omega}}\left\|\sqrt{\frac{h_{E}}{p_{E}}} \operatorname{Res}\left(u_{\ell}\right)\right\|_{L^{2}(E)}^{2}\right)^{1 / 2}
$$

for sufficiently small $0<\hat{\mu} \leq \hat{\mu}_{0}$, where $\hat{\mu}_{0}$ depends on $\alpha, \beta, b_{\infty}, c_{\infty}, \mu, C_{3}$, and $\rho$.

Proof. Observe that (6.12c) and (6.17) remain true if $u_{\ell+1}$ is replaced by $u$. Hence, we may also replace $u_{\ell+1}$ by $u$ in (6.14) and (6.20). By doing so, the combination of (6.14) and (6.20) yields after summing the squared norms over all $z \in \mathcal{N}_{\ell}^{1}$ the estimate

$$
\left(\sum_{E \in \mathcal{E}_{\Omega}}\left\|\sqrt{\frac{h_{E}}{p_{E}}} \operatorname{Res}\left(u_{\ell}\right)\right\|_{L^{2}(E)}^{2}\right)^{1 / 2} \leq C_{10}\left(\frac{p_{\ell}^{5 / 2}}{c_{\pi}}\left\|e_{\ell}\right\|_{\mathrm{PDE}}+\operatorname{Osc}\left(u_{\ell}\right)\right)
$$

where $C_{10}$ only depends $\alpha, \beta, b_{\infty}, c_{\infty}$, and $\rho$. The condition (6.23) implies (since $0 \leq c_{\pi} \leq 1$ (cf. Cor. 5.6))

$$
\operatorname{osc}\left(u_{\ell}\right) \leq \hat{\mu} \frac{1}{C_{10}} \frac{c_{\pi}}{p_{\ell}^{5 / 2}}\left(\sum_{E \in \mathcal{E}_{\Omega}}\left\|\sqrt{\frac{h_{E}}{p_{E}}} \operatorname{Res}\left(u_{\ell}\right)\right\|_{L^{2}(E)}^{2}\right)^{1 / 2} \leq \widehat{\mu}\left\|e_{\ell}\right\|_{\mathrm{PDE}}+\widehat{\mu} \operatorname{osc}\left(u_{\ell}\right) .
$$

For sufficiently small $0<\hat{\mu} \leq \hat{\mu}_{0}$, this implies (6.6). 
Corollary 6.5 Assume that the sequence of meshes and polynomial distributions are chosen such that the oscillation condition (6.6) holds on every level $\ell$. Let Conjecture 5.7 be satisfied. Then, the contraction of the error on level $\ell$ is given by

$$
\zeta_{\ell}:=\prod_{k=1}^{\ell}\left(1-\frac{C}{p_{k}^{5 / 2}}\right)
$$

for a constant $0<C \leq 1$ which is independent of the polynomial degrees, i.e.,

$$
\left\|e_{\ell}\right\|_{\mathrm{PDE}} \leq \zeta_{\ell}\left\|e_{0}\right\|_{\mathrm{PDE}} \text {. }
$$

Recall that $p_{k}$ denotes the maximal polynomial degree at level $k$ which is monotonously increasing. Define the sequence $\left(n_{i}\right)_{i \in \mathbb{N}}$ recursively by $n_{0}=0$ and, for $i=1,2, \ldots$, by the condition

$$
p_{k}=i \quad \text { for } n_{i-1}+1 \leq k \leq n_{i},
$$

i.e., the maximal polynomial degree stays fix for $\delta_{i}:=n_{i}-n_{i-1}$ consecutive levels.

1. If, for some $k_{0}>0$, it holds $n_{k}=\infty$ for all $k \geq k_{0}$, then $\zeta_{\ell} \leq\left(1-C p_{k_{0}}^{-5 / 2}\right)^{\ell} \rightarrow 0$ as $\ell \rightarrow \infty$.

2. If, $\sum_{k=1}^{\infty} p_{k}^{-5 / 2}=+\infty$, then $\lim _{\ell \rightarrow \infty} \zeta_{\ell}=0$.

3. If $\delta_{i} \geq c i^{3 / 2}$, then $\lim _{\ell \rightarrow \infty} \zeta_{\ell}=0$ as $\ell \rightarrow \infty$, while $\lim _{k \rightarrow \infty} p_{k}=\infty$.

Proof. The first statement is trivial. For the second statement we employ for $s>0$ and $C<1$

$$
\zeta_{\ell} \leq \exp \left(\sum_{k=1}^{\ell} \log \left(1-\frac{C}{p_{k}^{s}}\right)\right) .
$$

Note that, for $0<\varepsilon<1$,

$$
\log (1-\varepsilon) \leq-\varepsilon
$$

so that

$$
\zeta_{\ell} \leq \exp \left(-C \sum_{k=1}^{\ell} \frac{1}{p_{k}^{s}}\right) .
$$

From this, the second statement follows. For the third one we use

$$
\lim _{\ell \rightarrow \infty} \zeta_{\ell} \leq \exp \left(-C \sum_{i=1}^{\infty} \frac{\delta_{i}}{i^{s}}\right) .
$$

Hence, for $\delta_{i} \geq c i^{s-1}$ we have $\lim _{\ell \rightarrow \infty} \zeta_{\ell}=0$.

\section{A Lower Bound for the Constant $c_{\pi}$. Numerical Exper- iments}

In this appendix we will investigate the dependence of the stability constant $c_{\pi}$ of the polynomial projection operator $\Pi_{z}$ (cf. (5.8)) on the polynomial degree $p$. We consider mainly two cases: pure $p$-refinement and $h$-refinement. 


\section{A.1 $p$-Refinement}

First, we will rewrite the definition of $c_{\pi}$ as an algebraic eigenvalue problem which we will solve numerically. We have performed numerical experiments for the two-dimensional setting on stars as described in this paper but also considered the one-dimensional case where $\omega_{z}$ consists of the two intervals which have $z$ as a common endpoint.

\section{A.1.1 Equivalent Formulation}

The goal is to investigate the dependence of the constant

$$
c_{\pi}:=\inf _{v \in \mathbb{P}_{p}\left(\omega_{z}\right) \backslash\{0\}} \frac{\left(v, \Pi_{z}^{p} v\right)_{L^{2}\left(\omega_{z}\right)}}{\left\|\Phi_{z}^{1 / 2} v\right\|_{L^{2}\left(\omega_{z}\right)}^{2}}
$$

on the polynomial degree $p$ numerically. Let $d$ denote the spatial dimension. Let $\omega_{z}$ consists of $q \geq d$ simplices $K_{i}, 1 \leq i \leq q$.

By employing a global affine map we can pull back the star $\omega_{z}$ to a reference configuration, where $K_{1}=\widehat{K}$ is the unit simplex, on the expense that $c_{\pi}$ in (A.1) depends additionally on the shape regularity of $K_{1}$. Let $\chi_{i}: \widehat{K} \rightarrow K_{i}$ denote affine bijection with the special choice $\chi_{1}=\mathrm{id}$. Then,

$$
\left\|\Phi_{z}^{1 / 2} v\right\|_{L^{2}\left(\omega_{z}\right)}^{2}=\sum_{i=1}^{q} \frac{\left|K_{i}\right|}{|\widehat{K}|} \int_{\widehat{K}} \widehat{\Phi_{K}} \widehat{v}_{i}^{2}
$$

where $\widehat{v}_{i}=v \circ \chi_{i}$ and $\Phi_{\widehat{K}}$ denotes the product of barycentric coordinates. Let $\left(P_{n}\right)_{n \in \iota_{p}}$ denote a basis of $\mathbb{P}_{p}\left(\omega_{z}\right)$ for a suitable index set $\iota_{p}$. We write

$$
v=\sum_{n=0}^{p} v_{n} P_{n}
$$

and obtain

$$
\left\|\Phi_{z}^{1 / 2} v\right\|_{L^{2}\left(\omega_{z}\right)}^{2}=\mathbf{v}^{\top} \mathbf{M}^{(p)} \mathbf{v}
$$

where

$$
\left(\mathbf{M}_{i}^{(p)}\right)_{n, m}:=\frac{\left|K_{i}\right|}{|\widehat{K}|} \int_{\widehat{K}} \widehat{\Phi_{K}}\left(P_{n} \circ \chi_{i}\right)\left(P_{m} \circ \chi_{i}\right), \quad n, m \in \iota_{p} \quad \text { and } \quad \mathbf{M}^{(p)}:=\sum_{i=1}^{q} \mathbf{M}_{i}^{(p)} .
$$

For the special case that $\widehat{\Phi_{\widehat{K}}}$ is the polynomial bubble function we can choose an orthogonal basis for $\mathbb{P}_{p}(\widehat{K})$ (cf. [18], [12]) so that $\mathbf{M}_{1}^{(p)}$ is a diagonal matrix.

In order to invest (A.1) we introduce a matrix representation of $\Pi_{z}^{p} v$ with $v$ as in (A.2) via the ansatz

$$
\left.\Pi_{z}^{p} v\right|_{K_{i}}=\sum_{m \in \iota_{p}} w_{m, i} P_{m} \circ \chi_{i}^{-1}
$$

The coefficients $\mathbf{w}_{i}=\left(w_{m, i}\right)_{m \in \iota_{p}}$ are determined via

$$
\mathbf{w}_{i}=\left(\mathbf{M}_{1}^{(p-1)}\right)^{-1} \mathbf{W}_{i} \mathbf{v} \quad \text { with } \quad\left(\mathbf{W}_{i}\right)_{m, n}:=\frac{\left|K_{i}\right|}{|\widehat{K}|} \int_{\widehat{K}} \widehat{\Phi_{K}} P_{n} \circ \chi_{i} P_{m} \quad \text { for } \quad m \in \iota_{p-1}, n \in \iota_{p} .
$$


Hence,

$$
\left(v, \Pi_{z}^{p} v\right)_{z}=\mathbf{v}^{\top} \mathbf{B v} \quad \text { with } \quad \mathbf{B}:=\sum_{i=1}^{q} \mathbf{W}_{i}^{\top}\left(\mathbf{M}_{1,1}^{(p-1)}\right)^{-1} \mathbf{W}_{i}
$$

so that the constant $c_{\pi}$ has the algebraic representation

$$
c_{\pi}=\inf _{\mathbf{v} \in \mathbb{R}^{\iota p}} \frac{\mathbf{v}^{\top} \mathbf{B} \mathbf{v}}{\mathbf{v}^{\top} \mathbf{M}^{(p)} \mathbf{v}} .
$$

Hence, $c_{\pi}$ is the smallest eigenvalue of $\left(\mathbf{M}^{(p)}\right)^{-1 / 2} \mathbf{B}\left(\mathbf{M}^{(p)}\right)^{-1 / 2}$.

\section{A.1.2 The One-Dimensional Case}

In this case we have $\widehat{K}=[-1,1]$ and $P_{n}$ are the Jacobi polynomials $P_{n}^{(1,1)}$ which are defined as follows

$$
P_{n}^{(\alpha, \beta)}(x)=\frac{(2)_{n}}{n !}{ }_{2} F_{1}\left(\begin{array}{c}
-n, n+\alpha+\beta+1 \\
\alpha+1
\end{array} ; \frac{1-x}{2}\right),
$$

where $(\cdot)_{n}$ is Pochhammer's symbol and ${ }_{2} F_{1}$ is the terminating Gauss hypergeometric function

$$
{ }_{2} F_{1}\left(\begin{array}{c}
-n, b \\
c
\end{array} ; z\right)=\sum_{k=0}^{n} \frac{(-n)_{k}(b)_{k}}{(c)_{k} k !} z^{k} .
$$

We consider $K_{1}=\widehat{K}$ and $K_{2}=[1,1+\delta]$ for some $\delta>0$. Note that $\mathbf{M}_{1}^{(p)}$ in this case is given by

$$
\mathbf{M}_{1}^{(p)}=\operatorname{diag}\left[8 \frac{(n+1)}{(2 n+3)(n+2)}: n \in \iota_{p}\right] .
$$

The mapping $\chi_{2}$ is defined by

$$
\chi_{2}(\hat{x})=\frac{1-\hat{x}}{2}+\frac{1+\hat{x}}{2}(1+\delta) .
$$

To observe the behaviour of $c_{\pi}$ with respect to $p$ and $\delta$, we consider three different cases: $\delta=0.5, \delta=1, \delta=2, \delta=4$. The following observations can be obtained from Figure 4 :

- $c_{\pi}$ converges to a positive constant with respect to $p$,

- $c_{\pi}$ is properly bounded from below,

- $c_{\pi}$ is decreasing as $\delta$ goes to zero.

\section{A.1.3 The Two-Dimensional Case}

Now we consider Jacobi bivariate polynomials as our basis functions on the reference triangle, which are defined as follows:

$$
P_{n, k}^{1,1,1}(x, y):=(1-x)^{k} P_{n-k}^{(1,3+2 k)}(1-2 x) P_{k}^{(1,1)}\left(1-\frac{2 y}{1-x}\right),
$$




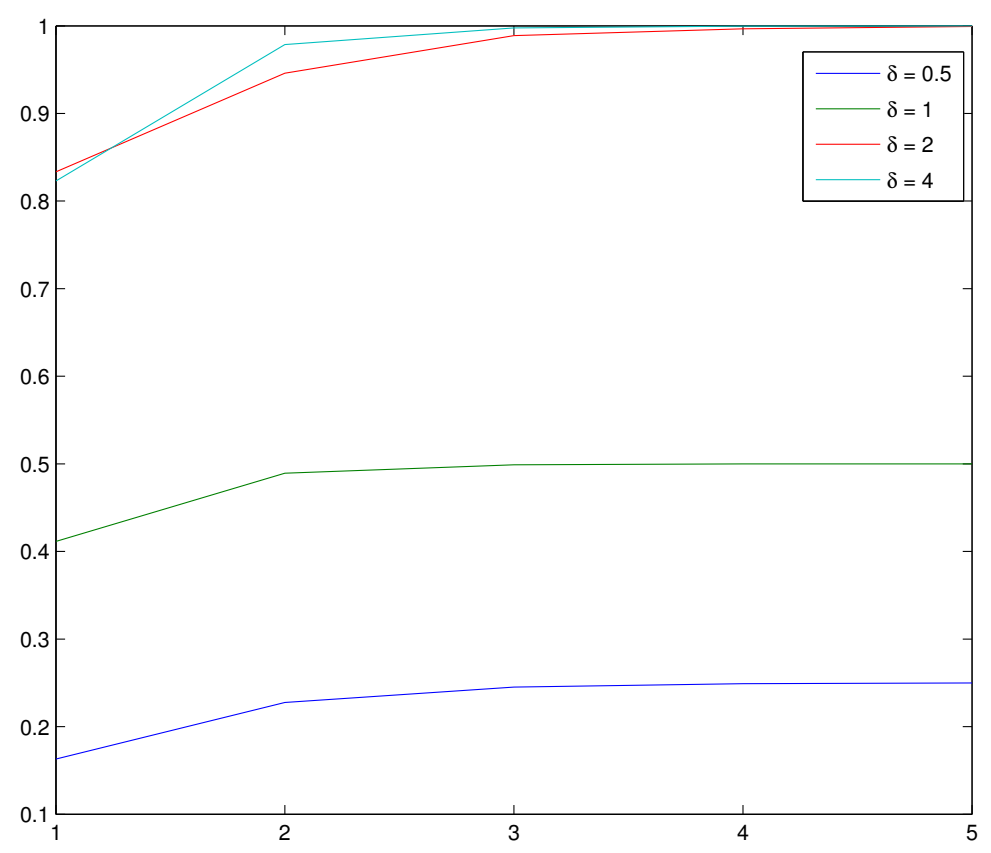

Figure 4: Performance of $c_{\pi}$ versus $p$ for the one-dimensional case.

which is a polynomial of degree $n$ in $x$ and $y$.

We study different triangulations. Again we assume that $K_{1}$ is the unit simplex and the common point of all triangles in the patch is $(0,0)$. The meshes consist of the following nodes and are illustrated in Figure 5:

$$
\begin{aligned}
& v_{1}=\{(0,0),(1,0),(0,1),(-1,1),(-1,0),(0,-1),(1,-1)\}, \\
& v_{2}=\{(0,0),(1,0),(0,1),(-1,1),(-2,0),(-2,-1),(-1,-3),(0,-3),(1,-1)\}, \\
& v_{3}=\{(0,0),(1,0),(0,1),(-1,1),(-3,0),(-4,-2),(-3,-3),(-1,-4),(0,-4),(1,-2)\}, \\
& v_{4}=\{(0,0),(1,0),(0,1),(-1,-1)\}, \\
& v_{5}=\{(0,0),(1,0),(0,1),(-0.1,-0.2)\}, \\
& v_{6}=\{(0,0),(1,0),(0,1),(-4,3),(-4,0),(-4,-4),(0,-4),(1,-0.1)\} .
\end{aligned}
$$

Figure 6 shows the behaviour of $c_{\pi}$ with respect to $p$ in each case and we summarize the main observations.

a. In the first three cases, i.e., the number of triangles (at least six) is varying while the shape regularity constant is always moderately bounded, the lower bound of $c_{\pi}$ is approximately 1 . It also shows that the constant $c_{\pi}$ is robust with respect to the elongation of the triangles which is in analogy to the one-dimensional observation $(\delta$ increases). 



Figure 5: Illustration of the geometric configuations described in (A.3).

b. If we consider the minimal number (three) elements, again, with moderate shape regular constant, we still get a proper lower bound. Recall that the dimension of the image space $\mathbb{P}_{p-1}\left(\mathcal{T}_{z}\right)$ (in (5.1)) increases with the number of triangles so that we expect that the constant $c_{\pi}$ becomes larger with increasing number of triangles.

c. On the other hand, if we consider the minimal configuration with only three triangles and large shape regularity constant (the area of the triangles is highly varying) as described by $v_{5}$, then the constant $c_{\pi}$ becomes smaller as expected.

d. Configuration $v_{6}$ supports the statement that, if the space $\mathbb{P}_{p-1}\left(\mathcal{T}_{z}\right)$ is large enough, then a few tiny elements can be still harmless. We can see that these numerical examples confirm our hypothesis that $c_{\pi}$ depends on the shape regularity of our meshes but does not depend on $p$.

\section{A.2 $h$-Refinement}

In this section we study the similar problem as in previous section but with $h$-refinement instead of $p$-refinement. In other word, we apply one level of regular $h$-refinement on each mesh and observe the behaviour of the constant $c_{\pi}$ with respect to $p$ on the refined mesh. To be able to make a comparison between the results, we take the same patches as in previous 


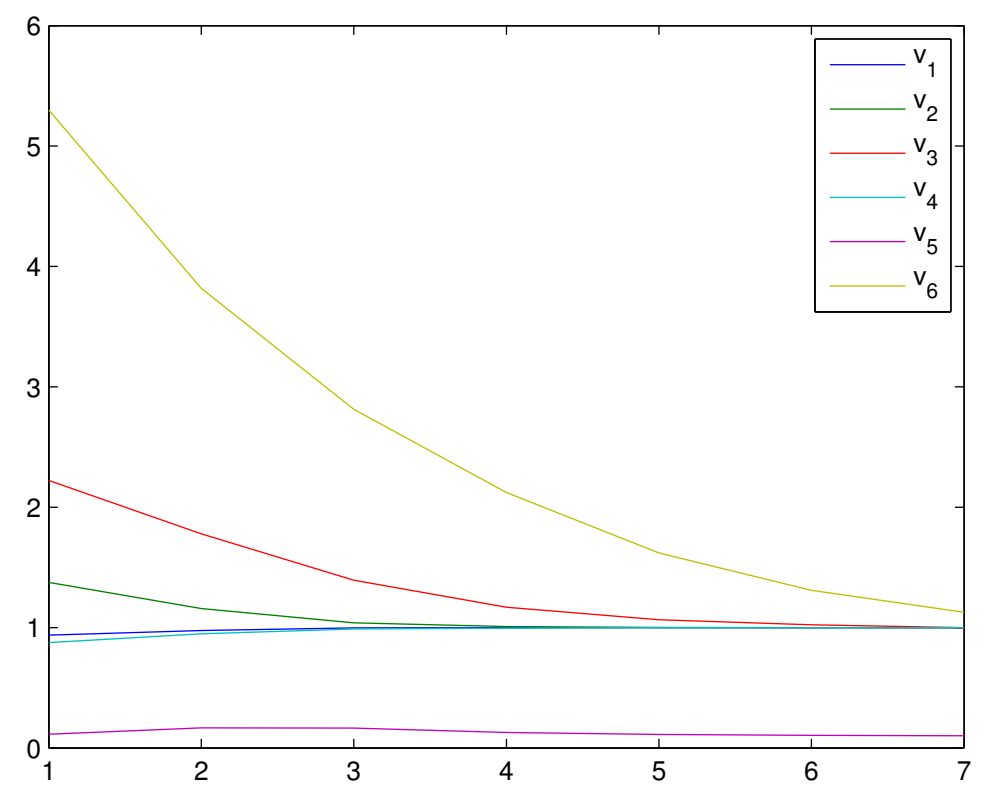

Figure 6: Performance of $c_{\pi}$ versus $p$ for the two-dimensional cases.

section. From the definition of $\Phi_{z}$ for this case we have

$$
c_{\pi}=\inf _{v \in \mathbb{P}_{p-1}(K)} \frac{\sum_{K \subset \omega_{z}} \int_{K} v \Phi_{K, K}^{(1)} v+\int_{K} v\left(\Phi_{K, K}^{(1)}+\Phi_{K}^{(2)}\right) \prod_{K}^{p-1} v}{\sum_{K \subset \omega_{z}} \int_{K} v\left(\Phi_{K, K}^{(1)}+\Phi_{K}^{(2)}\right) v}
$$

where $\Phi_{K, K}^{(1)}$ and $\Phi_{K}^{(2)}$ are piecewise linear and quadratic functions defined as in (5.4). Figure 7 shows the behaviour of $c_{\pi}$ for the same patches with respect to $p$. It supports our hypothesis and shows the similar behaviour as in $p$-version. Also here we observe that $c_{\pi}$ does not depend on $p$, but it only depends on the shape regularity of the mesh.

\section{B Polynomial Inverse Estimates}

We start with a one-dimensional estimate.

Lemma B.1 For $a<b$, let $\Phi_{[a, b]}(x)=\frac{(x-a)(b-x)}{(b-a)^{2}}$ denote the one-dimensional bubble function. Then,

$$
\left\|\left(\Phi_{[a, b]} v\right)^{\prime}\right\|_{L^{2}([a, b])} \leq C \frac{p+1}{b-a}\left\|\Phi_{[a, b]}^{1 / 2} v\right\|_{L^{2}([a, b])} \quad \forall v \in \mathbb{P}_{p}([a, b]) .
$$

Proof. We first prove the result for $(a, b)=(0,1)$. Observe that $\left\|\Phi_{[0,1]}^{\prime}\right\|_{L^{\infty}([0,1])}=1$ so that Leibniz rule gives us

$$
\left\|\left(\Phi_{[0,1]} v\right)^{\prime}\right\|_{L^{2}([0,1])} \leq\left\|\Phi_{[0,1]}^{\prime} v\right\|_{L^{2}([0,1])}+\left\|\Phi_{[0,1]} v^{\prime}\right\|_{L^{2}([0,1])} \leq\|v\|_{L^{2}([0,1])}+\left\|\Phi_{[0,1]} v^{\prime}\right\|_{L^{2}([0,1])} .
$$




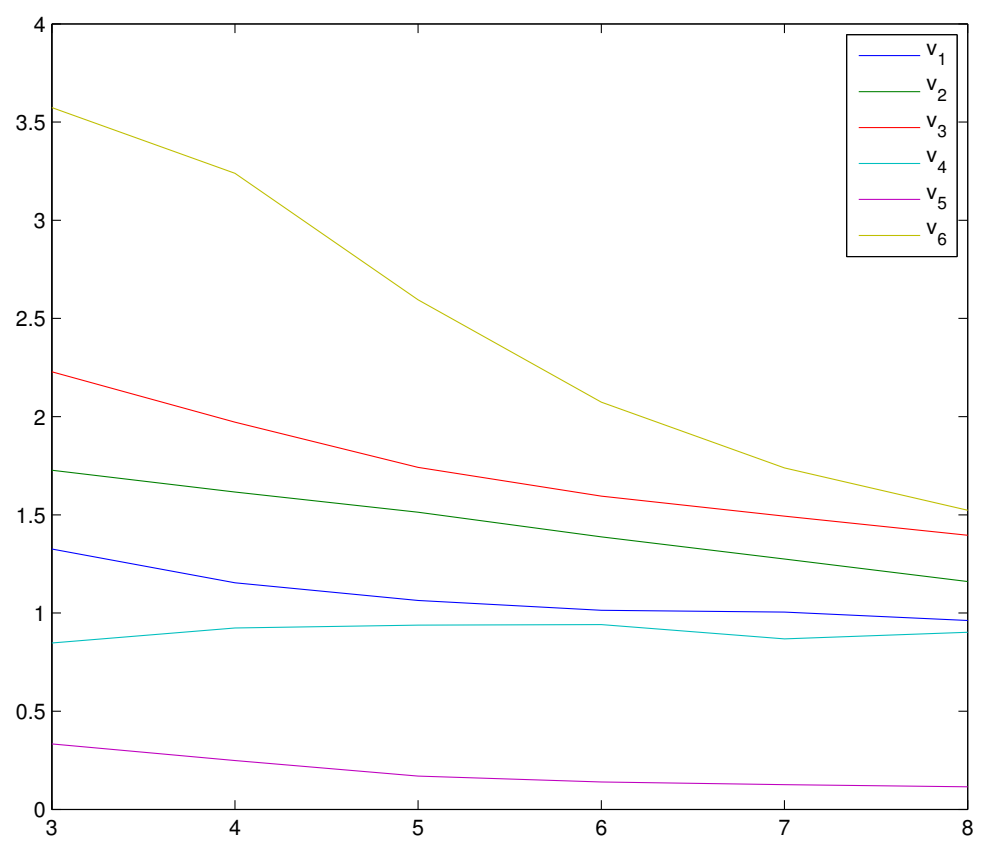

Figure 7: Behaviour of $c_{\pi}$ versus $p$ with one level of $h$-refinement

For the first term, we apply [16, Lemma 2.4 with $\alpha=0$ and $\beta=1]$ and for the second term [16, Lemma 2.4 with $\delta=1]$ to obtain

$$
\left\|\left(\Phi_{[0,1]} v\right)^{\prime}\right\|_{L^{2}([0,1])} \leq C(p+1)\left\|\Phi_{[0,1]}^{1 / 2} v\right\|_{L^{2}([0,1])} .
$$

The result then follows via a scaling argument.

Corollary B.2 Let $a<b$ and $\Phi_{[a, b]}$ be as in Lemma B.1. Let $\Psi_{[a, b]} \in W^{1, \infty}([a, b])$ be a function with the properties

$$
\left|\Psi_{[a, b]}\right| \leq C_{11} \Phi_{[a, b]} \quad \text { pointwise } \quad \text { and } \quad\left\|\Psi_{[a, b]}^{\prime}\right\|_{L^{\infty}([a, b])} \leq \frac{C_{12}}{b-a} .
$$

Then

$$
\left\|\left(\Psi_{[a, b]} v\right)^{\prime}\right\|_{L^{2}([a, b])} \leq C\left(C_{11}+C_{12}\right) \frac{p+1}{b-a}\left\|\Phi_{[a, b]}^{1 / 2} v\right\|_{L^{2}([a, b])} \quad \forall v \in \mathbb{P}_{p}([a, b]) .
$$

Proof. Leibniz' rule gives us

$$
\begin{aligned}
\left\|\left(\Psi_{[a, b]} v\right)^{\prime}\right\|_{L^{2}([a, b])} & \leq\left\|\Psi_{[a, b]}^{\prime} v\right\|_{L^{2}([a, b])}+\left\|\Psi_{[a, b]} v^{\prime}\right\|_{L^{2}([a, b])} \leq \frac{C_{12}}{b-a}\|v\|_{L^{2}([a, b])}+C_{11}\left\|\Phi_{[a, b]} v^{\prime}\right\|_{L^{2}([a, b])} \\
& \leq C\left(C_{11}+C_{12}\right) \frac{p+1}{b-a}\left\|\Phi_{[a, b]}^{1 / 2} v\right\|_{L^{2}([a, b])},
\end{aligned}
$$

where the last inequality follows as (B.1).

The two-dimensional version is formulated next. The estimates are similar to those in [23, Sec. 3.6] but differ by powers of the weight functions in the right-hand side and also by the choice of the weight function in Lemma B.4. The proofs follow the lines of the proofs in [23, Prop. 3.46] and also employs tools from [17, Appendix D]. 
Lemma B.3 Let $K$ denote a triangle and let $\Phi_{K}$ be the cubic bubble function as defined in (5.3). Then, it holds for all $v \in \mathbb{P}_{p}(K)$

$$
\left\|\nabla\left(\Phi_{K} v\right)\right\|_{L^{2}(K)} \leq C \frac{p+1}{h_{K}}\left\|\Phi_{K}^{1 / 2} v\right\|_{L^{2}(K)} .
$$

Proof. Let $K=\widehat{K}$ be the two-dimensional reference triangle. Note that

$$
\Phi_{\widehat{K}}\left(x_{1}, x_{2}\right)=\Phi_{\left[0,1-x_{1}\right]}\left(x_{2}\right)\left(1-x_{1}\right) \Phi_{[0,1]}\left(x_{1}\right)
$$

with $\Phi_{[a, b]}$ as in Lemma B.1. First, we consider the derivative with respect to $x_{2}$ and obtain

$$
\begin{aligned}
\left\|\partial_{2}\left(\Phi_{\widehat{K}} v\right)\right\|_{L^{2}(\widehat{K})}^{2} & =\int_{0}^{1}\left(\int_{0}^{1-x_{1}}\left(\partial_{2}\left(\Phi_{\widehat{K}}\left(x_{1}, x_{2}\right) v\left(x_{1}, x_{2}\right)\right)\right)^{2} d x_{2}\right) d x_{1} \\
& =\int_{0}^{1} \Phi_{[0,1]}^{2}\left(x_{1}\right)\left(1-x_{1}\right)^{2}\left(\int_{0}^{1-x_{1}}\left(\frac{\partial}{\partial x_{2}}\left(\Phi_{\left[0,1-x_{1}\right]}\left(x_{2}\right) v\left(x_{1}, x_{2}\right)\right)\right)^{2} d x_{2}\right) d x_{1} .
\end{aligned}
$$

We then get

$$
\begin{aligned}
& \left(1-x_{1}\right)^{2} \int_{0}^{1-x_{1}}\left(\frac{\partial}{\partial x_{2}}\left(\Phi_{\left[0,1-x_{1}\right]}\left(x_{2}\right) v\left(x_{1}, x_{2}\right)\right)\right)^{2} d x_{2} \\
& =\left(1-x_{1}\right)^{2}\left\|\left(\Phi_{\left[0,1-x_{1}\right]} v\left(x_{1}, \cdot\right)\right)^{\prime}\right\|_{L^{2}\left(0,1-x_{1}\right)}^{2} \\
& \stackrel{\text { Lem. B.1 }}{\leq} C(p+1)^{2}\left\|\Phi_{\left[0,1-x_{1}\right]}^{1 / 2} v\left(x_{1}, \cdot\right)\right\|_{L^{2}\left(0,1-x_{1}\right)}^{2} .
\end{aligned}
$$

Since $\Phi_{[0,1]}^{2}\left(x_{1}\right) \Phi_{\left[0,1-x_{1}\right]}\left(x_{2}\right) \leq \Phi_{\widehat{K}}\left(x_{1}, x_{2}\right)$ we end up with

$$
\begin{aligned}
\left\|\partial_{2}\left(\Phi_{\widehat{K}} v\right)\right\|_{L^{2}(\widehat{K})}^{2} & \leq C(p+1)^{2} \int_{0}^{1} \int_{0}^{1-x_{1}} \Phi_{[0,1]}^{2}\left(x_{1}\right) \Phi_{\left[0,1-x_{1}\right]}\left(x_{2}\right) v^{2}\left(x_{1}, x_{2}\right) d x_{2} d x_{1} \\
& \leq C(p+1)^{2}\left\|\Phi_{\widehat{K}}^{1 / 2} v\right\|_{L^{2}(\widehat{K})}^{2} .
\end{aligned}
$$

Since $\widehat{K}, \psi_{\widehat{K}}$, and the integral are invariant under permutations of the coordinates, the same estimate holds for the other partial derivatives.

Lemma B.4 Let $K$ be regularly h-refined and let $\Phi_{K}^{(2)}$ be as explained in Definition 5.1 and illustrated in Figure 3. Then, it holds for all $v \in \mathbb{P}_{p}(K)$

$$
\left\|\nabla\left(\Phi_{K}^{(2)} v\right)\right\|_{L^{2}(K)} \leq C \frac{p+1}{h_{K}}\left\|\sqrt{\Phi_{K}^{(2)}} v\right\|_{L^{2}(K)} .
$$

Proof. Via an affine transformation it suffices to prove the result for the reference element $K_{1}=\widehat{K}$ and $K_{2}=\operatorname{conv}\left\{\left(\begin{array}{l}0 \\ 0\end{array}\right),\left(\begin{array}{l}0 \\ 1\end{array}\right),\left(\begin{array}{c}-1 \\ 0\end{array}\right)\right\}$. The common edge is $E=\{0\} \times(0,1)$. Let $K=$ $K_{1} \cup K_{2}$. The edge bubble $\Phi_{K}^{(2)}($ cf. (5.4)) is given by

$$
\Phi_{K}^{(2)}\left(x_{1}, x_{2}\right)=x_{2}\left(1-\left|x_{1}\right|-x_{2}\right) .
$$


We first consider the derivative with respect to $x_{2}$. Let

$$
\Phi_{\left[0,1-\left|x_{1}\right|\right]}\left(x_{2}\right)=\frac{x_{2}\left(1-\left|x_{1}\right|-x_{2}\right)}{\left(1-\left|x_{1}\right|\right)^{2}},
$$

i.e., $\Phi_{\left[0,1-\left|x_{1}\right|\right]}$ is the one-dimensional bubble function for $\left[0,1-\left|x_{1}\right|\right]$ and satisfies $\Phi_{K}^{(2)}=$ $\left(1-\left|x_{1}\right|\right)^{2} \Phi_{\left[0,1-\left|x_{1}\right|\right]}$. Hence,

$$
\begin{aligned}
\left\|\partial_{2}\left(\Phi_{K}^{(2)} v\right)\right\|_{L^{2}(K)}^{2} & =\int_{-1}^{1}\left(1-\left|x_{1}\right|\right)^{4} \int_{0}^{1-\left|x_{1}\right|}\left(\partial_{2}\left(\Phi_{\left[0,1-\left|x_{1}\right|\right]}\left(x_{2}\right) v\left(x_{1}, x_{2}\right)\right)\right)^{2} d x_{2} d x_{1} \\
& \stackrel{\text { Lem. B.1 }}{\leq} C(p+1)^{2} \int_{-1}^{1}\left(1-\left|x_{1}\right|\right)^{2} \int_{0}^{1-\left|x_{1}\right|} \Phi_{\left[0,1-\left|x_{1}\right|\right]}\left(x_{2}\right) v^{2}\left(x_{1}, x_{2}\right) d x_{2} d x_{1} \\
& =C(p+1)^{2} \int_{K} \Phi_{K}^{(2)} v^{2} .
\end{aligned}
$$

Next, we will estimate the derivative with respect to $x_{1}$. We split the triangle into the two regions

$$
D_{1}:=\left\{\left(\begin{array}{c}
x_{1} \\
x_{2}
\end{array}\right) \in K: x_{2} \leq \frac{1}{2}\right\} \quad \text { and } \quad D_{2}:=\operatorname{conv}\left\{\left(\begin{array}{c}
1 / 2 \\
1 / 2
\end{array}\right),\left(\begin{array}{l}
0 \\
1
\end{array}\right),\left(\begin{array}{c}
-1 / 2 \\
1 / 2
\end{array}\right)\right\} .
$$

In addition, we will need

$$
D_{3}:=\operatorname{conv}\left\{\left(\begin{array}{l}
0 \\
0
\end{array}\right),\left(\begin{array}{l}
1 / 2 \\
1 / 2
\end{array}\right),\left(\begin{array}{l}
0 \\
1
\end{array}\right),\left(\begin{array}{c}
-1 / 2 \\
1 / 2
\end{array}\right)\right\}
$$

On $D_{1}$ we obtain

$$
\begin{aligned}
\left\|\partial_{1}\left(\Phi_{K}^{(2)} v\right)\right\|_{L^{2}\left(D_{1}\right)}^{2} & \leq \int_{0}^{1 / 2} x_{2}^{2}\left(1-x_{2}\right)^{2} \int_{x_{2}-1}^{1-x_{2}}\left(\partial_{1}\left(\frac{1-\left|x_{1}\right|-x_{2}}{1-x_{2}} v\left(x_{1}, x_{2}\right)\right)\right)^{2} d x_{1} d x_{2} \\
& \stackrel{\text { Lem. B. } 2}{\leq} C(p+1)^{2} \int_{0}^{1 / 2} \int_{x_{2}-1}^{1-x_{2}} \frac{x_{2}}{1-x_{2}} \Phi_{K}^{(2)}\left(x_{1}, x_{2}\right) v^{2}\left(x_{1}, x_{2}\right) d x_{1} d x_{2} \\
& \leq C(p+1)^{2}\left\|\sqrt{\Phi_{K}^{(2)}} v\right\|_{L^{2}\left(D_{1}\right)}^{2},
\end{aligned}
$$

since $x_{2} /\left(1-x_{2}\right) \leq 1$ on $D_{1}$.

On $D_{2}$, we observe that

$$
\frac{1}{2}\left\|\partial_{1}\left(\Phi_{K}^{(2)} v\right)\right\|_{L^{2}\left(D_{2}\right)}^{2} \leq\left\|v \partial_{1} \Phi_{K}^{(2)}\right\|_{L^{2}\left(D_{2}\right)}^{2}+\left\|\Phi_{K}^{(2)} \partial_{1} v\right\|_{L^{2}\left(D_{2}\right)}^{2} .
$$

Let $d: D_{3} \rightarrow \mathbb{R}$ be defined by $d\left(x_{1}, x_{2}\right)=c_{3}$ dist $\left(\left(x_{1}, x_{2}\right)^{\top}, \partial D_{3}\right)$ where the scaling $c_{3}$ is chosen such that $d$ interpolates $\Phi_{K}^{(2)}$ at the vertices of the two triangles $K_{m} \cap D_{3}, m=1,2$. Note that

$$
d \leq \Phi_{K}^{(2)} \leq 1 \quad \text { pointwise in } D_{3} \text { and } \Phi_{K}^{(2)} \leq 2 d \text { pointwise in } D_{2} .
$$

Since $\left\|\partial_{1} \Phi_{K}^{(2)}\right\|_{L^{\infty}(K)} \leq C$ we obtain for the first term in (B.4) as in (5.16b)

$$
\left\|v \partial_{1} \Phi_{K}^{(2)}\right\|_{L^{2}\left(D_{2}\right)} \leq C\|v\|_{L^{2}\left(D_{2}\right)} \leq C\|v\|_{L^{2}\left(D_{3}\right)} \leq C(p+1)\left\|d^{1 / 2} v\right\|_{L^{2}\left(D_{3}\right)} \leq C(p+1)\left\|\sqrt{\Phi_{K}^{(2)}} v\right\|_{L^{2}\left(D_{3}\right)} .
$$




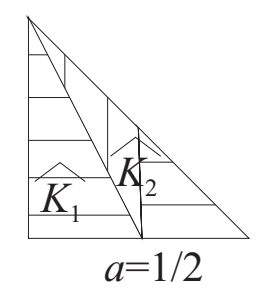

Figure 8: Reference triangle $\widehat{K}$ which is split into $\widehat{K}_{1}$ and $\widehat{K}_{2}$. The shaded regions illustrate the integration domains in the splitting of the integral in (B.8).

For the second term in (B.4) we get, again, as in (5.16b)

$$
\left\|\Phi_{K}^{(2)} \partial_{1} v\right\|_{L^{2}\left(D_{2}\right)} \leq 2\left\|d \partial_{1} v\right\|_{L^{2}\left(D_{3}\right)} \stackrel{[16,(23) \text { with } \delta=1]}{\leq} C(p+1)\|\sqrt{d} v\|_{L^{2}\left(D_{3}\right)} \leq C(p+1)\left\|\sqrt{\Phi_{K}^{(2)}} v\right\|_{L^{2}\left(D_{3}\right)} .
$$

The combination of (B.4) and (B.5) yields

$$
\left\|\partial_{1}\left(\Phi_{K}^{(2)} v\right)\right\|_{L^{2}\left(D_{2}\right)}^{2} \leq C(p+1)^{2}\left\|\sqrt{\Phi_{K}^{(2)}} v\right\|_{L^{2}\left(D_{3}\right)}^{2} .
$$

The combination of (B.2), (B.3), and (B.6) yields the assertion.

The following lemma is illustrated in Figure 8.

Lemma B.5 Let $\widehat{K}$ be the reference triangle split into $\widehat{K}_{1}=\operatorname{conv}\left(\left(\begin{array}{l}0 \\ 0\end{array}\right),\left(\begin{array}{l}a \\ 0\end{array}\right),\left(\begin{array}{l}0 \\ 1\end{array}\right)\right)$ and $\widehat{K}_{2}=$ conv $\left(\left(\begin{array}{l}a \\ 0\end{array}\right),\left(\begin{array}{l}1 \\ 0\end{array}\right),\left(\begin{array}{l}0 \\ 1\end{array}\right)\right)$ for some $\left.a \in\right] 0,1\left[\right.$. Let $\varphi_{E}^{\text {lin }}$ denote the continuous, piecewise linear function which has value 1 at $\left(\begin{array}{l}a \\ 0\end{array}\right)$ and vanishes at $\partial \widehat{K} \backslash E_{1}$ with $E_{1}=[0,1] \times\{0\}$. Then, for any polynomial $v \in \mathbb{P}_{p}$ which is constant with respect to $x_{2}$ it holds

$$
\begin{gathered}
\left\|\sqrt{\varphi_{E}^{\operatorname{lin}}} v\right\|_{L^{2}(\widehat{K})} \leq C\left\|\sqrt{\varphi_{E}^{\operatorname{lin}}} v\right\|_{L^{2}\left(E_{1}\right)}, \\
\left\|\nabla\left(\varphi_{E}^{\operatorname{lin}} v\right)\right\|_{L^{2}(\widehat{K})} \leq C(p+1)\left\|\sqrt{\varphi_{E}^{\operatorname{lin}} v}\right\|_{L^{2}\left(E_{1}\right)} .
\end{gathered}
$$

Proof. We prove this lemma only for $a=1 / 2$ to reduce technicalities. The arguments apply verbatim for the general case. The function $\varphi_{E}^{\text {lin }}$ and its partial derivatives are given by

$$
\begin{aligned}
\varphi_{E}^{\operatorname{lin}}\left(x_{1}, x_{2}\right) & =\left\{\begin{array}{ll}
2 x_{1} & \left(x_{1}, x_{2}\right) \in \widehat{K}_{1}, \\
2\left(1-x_{1}-x_{2}\right) & \left(x_{1}, x_{2}\right) \in \widehat{K}_{2},
\end{array} \quad \partial_{2} \varphi_{E}^{\operatorname{lin}}\left(x_{1}, x_{2}\right)= \begin{cases}0 & \left(x_{1}, x_{2}\right) \in \widehat{K}_{1}, \\
-2 & \left(x_{1}, x_{2}\right) \in \widehat{K}_{2},\end{cases} \right. \\
\partial_{1} \varphi_{E}^{\operatorname{lin}}\left(x_{1}, x_{2}\right) & = \begin{cases}2 & \left(x_{1}, x_{2}\right) \in \widehat{K}_{1}, \\
-2 & \left(x_{1}, x_{2}\right) \in \widehat{K}_{2} .\end{cases}
\end{aligned}
$$

Since $v \in \mathbb{P}_{p}$ is constant with respect to $x_{2}$ we write, with a slight abuse of notation, $v\left(x_{1}, x_{2}\right)=$ $v\left(x_{1}\right)$. Hence,

$$
\left\|\sqrt{\varphi_{E}^{\operatorname{lin}}} v\right\|_{L^{2}(\widehat{K})}^{2}=\int_{0}^{1} v^{2}\left(x_{1}\right)\left(\int_{0}^{1-x_{1}} \varphi_{E}^{\operatorname{lin}}\left(x_{1}, x_{2}\right) d x_{2}\right) d x_{1}
$$


The result of the inner integration is

$$
\begin{aligned}
r\left(x_{1}\right) & :=\int_{0}^{1-x_{1}} \varphi_{E}^{\operatorname{lin}}\left(x_{1}, x_{2}\right) d x_{2}=\left\{\begin{array}{l}
2\left(\int_{0}^{1-2 x_{1}} x_{1} d x_{2}+\int_{1-2 x_{1}}^{1-x_{1}}\left(1-x_{1}-x_{2}\right) d x_{2}\right) \\
2 \int_{0}^{1-x_{1}}\left(1-x_{1}-x_{2}\right) d x_{2}
\end{array}\right. \\
& = \begin{cases}x_{1}\left(2-3 x_{1}\right) & x_{1} \leq 1 / 2 \\
\left(1-x_{1}\right)^{2} & x_{1}>1 / 2 .\end{cases}
\end{aligned}
$$

Since $r \leq \varphi_{E}^{\operatorname{lin}}(\cdot, 0)$ pointwise on $[0,1]$, the first assertion follows.

Next, we investigate the derivative with respect to $x_{2}$. It holds

$$
\partial_{2}\left(\varphi_{E}^{\operatorname{lin}} v\right)=v \partial_{2} \varphi_{E}^{\operatorname{lin}}=v \times \begin{cases}0 & \left(x_{1}, x_{2}\right) \in \widehat{K}_{1} \\ -2 & \left(x_{1}, x_{2}\right) \in \widehat{K}_{2}\end{cases}
$$

Thus,

$$
\begin{aligned}
\int_{\widehat{K}}\left(\partial_{2}\left(\varphi_{E}^{\operatorname{lin}} v\right)\right)^{2} & =\int_{\widehat{K}}\left(v \partial_{2} \varphi_{E}^{\operatorname{lin}}\right)^{2} \leq 4 \int_{\widehat{K}} v^{2} \leq 4 \int_{0}^{1} v^{2} \\
& \leq 16, \text { Lemma 2.4 with } \alpha=0 \text { and } \beta=1] \\
\leq & 4(p+1)^{2} \int_{0}^{1} \Phi_{[0,1]} v^{2} \leq 4 C(p+1)^{2} \int_{0}^{1} \varphi_{E}^{\operatorname{lin}} v^{2} .
\end{aligned}
$$

For the derivative with respect to $x_{1}$, we get

$$
q\left(x_{1}, x_{2}\right):=\partial_{1}\left(\varphi_{E}^{\operatorname{lin}}\left(x_{1}, x_{2}\right) v\left(x_{1}\right)\right)=2 \begin{cases}\left(x_{1} v\left(x_{1}\right)\right)^{\prime} & \text { in } \widehat{K}_{1}, \\ \left(1-x_{1}-x_{2}\right) v^{\prime}\left(x_{1}\right)-v\left(x_{1}\right) & \text { in } \widehat{K}_{2} .\end{cases}
$$

The function $q$ is on $\widehat{K}_{1}$ and on $\widehat{K}_{2}$, an affine function with respect to $x_{2}$. We split the integral into

$$
\int_{0}^{1} \int_{0}^{1-x_{1}} \ldots=\int_{0}^{1 / 2} \int_{0}^{1-2 x_{1}} \ldots+\int_{0}^{1 / 2} \int_{1-2 x_{1}}^{1-x_{1}} \ldots+\int_{1 / 2}^{1} \int_{0}^{1-x_{1}}=: W_{1}+W_{2}+W_{3}
$$

and obtain for the summands

$$
\begin{aligned}
W_{1} & =4 \int_{0}^{1 / 2}\left(1-2 x_{1}\right)\left(\left(x_{1} v\left(x_{1}\right)\right)^{\prime}\right)^{2} d x_{1} \leq \int_{0}^{1 / 2}\left(\left(2 x_{1} v\left(x_{1}\right)\right)^{\prime}\right)^{2} d x_{1} \\
& \leq \int_{0}^{1}\left(\left(\varphi_{E}^{\operatorname{lin}}\left(x_{1}, 0\right) v\left(x_{1}\right)\right)^{\prime}\right)^{2} d x_{1} \stackrel{\text { Cor. B. } 2}{\leq} C(p+1)^{2}\left\|\sqrt{\varphi_{E}^{\operatorname{lin}}} v\right\|_{L^{2}([0,1])}^{2}
\end{aligned}
$$


For $W_{2}$ and $W_{3}$ we use the fact that the Simpson rule is exact for quadratic polynomials and (B.7) to obtain

$$
\begin{aligned}
W_{2} & =4 \int_{0}^{1 / 2} \int_{1-2 x_{1}}^{1-x_{1}}\left(\left(1-x_{1}-x_{2}\right) v^{\prime}\left(x_{1}\right)-v\left(x_{1}\right)\right)^{2} d x_{2} d x_{1} \\
& =\frac{2}{3} \int_{0}^{1 / 2} x_{1}\left((\underbrace{x_{1} v^{\prime}\left(x_{1}\right)-v\left(x_{1}\right)}_{=\left(x_{1} v\left(x_{1}\right)\right)^{\prime}-2 v\left(x_{1}\right)})^{2}+4(\underbrace{\frac{x_{1}}{2} v^{\prime}\left(x_{1}\right)-v\left(x_{1}\right)}_{\frac{\left(x_{1} v\left(x_{1}\right)\right)^{\prime}}{2}-\frac{3}{2} v\left(x_{1}\right)})^{2}+v^{2}\left(x_{1}\right)\right) d x_{1} \\
& \leq \frac{2}{3} \int_{0}^{1 / 2} x_{1}\left(4\left(\left(x_{1} v\left(x_{1}\right)\right)^{\prime}\right)^{2}+27 v^{2}\left(x_{1}\right)\right) d x_{1} \quad \text { Cor. B.2 } C(p+1)^{2}\left\|\sqrt{\varphi_{E}^{\operatorname{lin}}} v\right\|_{L^{2}([0,1])}^{2} .
\end{aligned}
$$

Finally, for $W_{3}$ we obtain

$$
\begin{aligned}
W_{3} & =4 \int_{1 / 2}^{1} \int_{0}^{1-x_{1}}\left(\left(1-x_{1}-x_{2}\right) v^{\prime}\left(x_{1}\right)-v\left(x_{1}\right)\right)^{2} d x_{2} d x_{1} \\
& =4 \int_{1 / 2}^{1} \frac{1-x_{1}}{6}\left((\underbrace{\left(1-x_{1}\right) v^{\prime}\left(x_{1}\right)-v\left(x_{1}\right)}_{\left(\left(1-x_{1}\right) v\left(x_{1}\right)\right)^{\prime}})^{2}+4(\underbrace{\left(\frac{1-x_{1}}{2}\right) v^{\prime}\left(x_{1}\right)-v\left(x_{1}\right)}_{\frac{1}{2}\left(\left(\left(1-x_{1}\right) v\left(x_{1}\right)\right)^{\prime}-v\left(x_{1}\right)\right)})^{2}+v^{2}\left(x_{1}\right)\right) d x_{1} \\
& 4 \int_{1 / 2}^{1} \frac{1-x_{1}}{6}\left(3\left(\left(\left(1-x_{1}\right) v\left(x_{1}\right)\right)^{\prime}\right)^{2}+9 v\left(x_{1}\right)\right) d x_{1} \\
& \leq \frac{1}{12} \int_{1 / 2}^{1} 3\left(\left(2\left(1-x_{1}\right) v\left(x_{1}\right)\right)^{\prime}\right)^{2}+36 v^{2}\left(x_{1}\right) d x_{1} \leq \frac{1}{4} \int_{0}^{1}\left(\left(\varphi_{E}^{\operatorname{lin}}\left(x_{1}\right) v\left(x_{1}\right)\right)^{\prime}\right)^{2} d x_{1}+3\|v\|_{L^{2}[0,1]}^{2} \\
& \quad \text { Cor. B.2 } C(p+1)^{2}\left\|\sqrt{\varphi_{E}^{\operatorname{lin}}} v\right\|_{L^{2}([0,1])}^{2} .
\end{aligned}
$$

Corollary B.6 Let $\widehat{K}$ be the reference triangle and let $\varphi_{E}\left(x_{1}, x_{2}\right)=x_{1}\left(1-x_{1}-x_{2}\right)$ denote the quadratic edge bubble on $\widehat{K}$ for the edge $E_{1}=[0,1] \times\{0\}$. Then, for any polynomial $v \in \mathbb{P}_{p}$ which is constant with respect to $x_{2}$ it holds

$$
\begin{aligned}
\left\|\sqrt{\varphi_{E}} v\right\|_{L^{2}(\widehat{K})} & \leq C\left\|\sqrt{\varphi_{E}} v\right\|_{L^{2}\left(E_{1}\right)}, \\
\left\|\nabla\left(\varphi_{E} v\right)\right\|_{L^{2}(\widehat{K})} & \leq C(p+1)\left\|\sqrt{\varphi_{E}} v\right\|_{L^{2}\left(E_{1}\right)} .
\end{aligned}
$$

The proof follows by a simple repetition of the arguments of the proof of Lemma B.5.

Lemma B.7 Let $K$ be a triangle and $E$ one of its edges. Then, for any of the functions $\varphi_{E}$ (6.4) and corresponding version $\Phi_{K}$ as in (5.4) it holds

$$
\left\|\varphi_{E}^{1 / 2} v\right\|_{L^{2}(D)} \leq C(p+1)\left\|\Phi_{K}^{1 / 2} v\right\|_{L^{2}(D)} \quad \forall v \in \mathbb{P}_{p}(D) .
$$


The proof requires two preparatory lemmata and follows the ideas in $[17$, Appendix D].

Lemma B.8 Let $I=[a, b]$ for some $a<b$ and let $\omega: I \rightarrow \mathbb{R}$ be a weight function which satisfies

$$
\exists A, B, D \geq 0 \text { with }\left\{\begin{array}{l}
\omega \text { is positive in }] a, b[ \\
\omega(x) \leq A \varphi_{a}(x)+B \varphi_{b}(x)+D \Phi_{[a, b]}(x),
\end{array}\right.
$$

where $\varphi_{b}(x)=\frac{x-a}{b-a}, \varphi_{a}=1-\varphi_{b}$, and $\Phi_{[a, b]}=\varphi_{a} \varphi_{b}$ as in (B.1). Then, it holds

$$
\left\|\omega^{1 / 2} v\right\|_{L^{2}(I)} \leq C(p+1)\left\|\sqrt{\omega \Phi_{[a, b]}} v\right\|_{L^{2}(I)} \quad \forall v \in \mathbb{P}_{p}(I),
$$

where $C$ is independent of $p, v, \omega, a, b$.

Proof. By employing an affine transform it is sufficient to prove the assertion for the unit interval $I=[0,1]$.

a) $\omega(x)=\Phi_{[0,1]}(x)=x(1-x)$. We may apply standard inverse estimates to obtain

$$
\left\|\Phi_{[0,1]}^{1 / 2} v\right\|_{L^{2}(I)} \stackrel{[16, \text { with } \alpha=1, \beta=2]}{\leq} C(p+1)\left\|\Phi_{[0,1]} v\right\|_{L^{2}(I)}=C(p+1)\left\|\sqrt{\omega \Phi_{[0,1]}} v\right\|_{L^{2}(I)} .
$$

b) For $\omega(x)=\varphi_{b}(x)=x$ we observe that $\omega(x) \leq 2 \Phi_{[0,1]}(x)$ holds for all $0 \leq x \leq 1 / 2$ so that

$$
\left\|\omega^{1 / 2} v\right\|_{L^{2}(I)}^{2} \leq 2\left\|\Phi_{[0,1]}^{1 / 2} v\right\|_{L^{2}(I)}^{2}+\|v\|_{L^{2}\left(\left[\frac{1}{2}, 1\right]\right)}^{2} \stackrel{[16, \text { Lem. 2.4] }}{\leq} C(p+1)^{2}\left(\left\|\Phi_{[0,1]} v\right\|_{L^{2}(I)}^{2}+\left\|\Phi_{\left[\frac{1}{2}, 1\right]}^{1 / 2} v\right\|_{L^{2}\left(\left[\frac{1}{2}, 1\right]\right)}^{2}\right) .
$$

The result now follows from $\Phi_{[0,1]}^{1 / 2} \leq \omega^{1 / 2}$ pointwise in $[0,1]$ and $\sqrt{2 \omega} \geq 1$ pointwise on $\left[\frac{1}{2}, 1\right]$.

c) The $\omega(x)=\varphi_{a}(x)$ follows from Case b by symmetry.

d) Let $\omega$ be a general weight function which satisfies the assumptions of the lemma. Hence, from Part a,b,c we conclude that

$\left\|\omega^{1 / 2} v\right\|_{L^{2}(I)}^{2}=A\left\|\sqrt{\varphi_{0}} v\right\|_{L^{2}(I)}^{2}+B\left\|\sqrt{\varphi_{1}} v\right\|_{L^{2}(I)}^{2}+D\left\|\sqrt{\Phi_{[0,1]}} v\right\|_{L^{2}(I)}^{2} \leq C^{\prime}(p+1)^{2}\left\|\sqrt{\omega \Phi_{[0,1]}} v\right\|_{L^{2}(I)}^{2}$ holds.

The following lemma is a weighted version of [17, Lem. D3].

Lemma B.9 Let $d \in(0,1), a, b$ be given such that $-1+a d<1+b d$ and define the trapezoid

$$
D:=D(a, b, d):=\left\{\left(x_{1}, x_{2}\right) \in \mathbb{R}^{2} \mid x_{2} \in(0, d) \quad \text { and } \quad-1+a x_{2}<x_{1}<1+b x_{2}\right\} .
$$

Let $\omega \in \mathbb{P}_{2}(D)$ be a polynomial such that for any $0 \leq x_{2} \leq d, \omega\left(\cdot, x_{2}\right)$ has property $P_{\left[-1+a x_{2}, 1+b x_{2}\right]}$.

On $D$ we define the weight function

$$
\Phi_{a, b, d}\left(x_{1}, x_{2}\right):=\min \left\{\left|x_{1}-\left(-1+a x_{2}\right)\right|,\left|x_{1}-\left(1+b x_{2}\right)\right|\right\}
$$

which measures the distance of the point $\left(x_{1}, x_{2}\right)$ from the lateral edges of $D$. Then, there exists a constant $C=C(a, b, d)$ such that for all $p \in \mathbb{N}$ and all polynomials $v \in \mathbb{P}_{p}(D)$ it holds

$$
\left\|\omega^{1 / 2} v\right\|_{L^{2}(D)} \leq C(p+1)\left\|\sqrt{\omega \Phi_{a, b, d}} v\right\|_{L^{2}(D)} .
$$


Proof. Note that

$$
C_{13} \Phi_{\left[-1+a x_{2}, 1+b x_{2}\right]}\left(x_{1}\right) \leq \Phi_{a, b, d}\left(x_{1}, x_{2}\right) \leq C_{14} \Phi_{\left[-1+a x_{2}, 1+b x_{2}\right]}\left(x_{1}\right)
$$

for positive constants $C_{13}, C_{14}$ which only depends on $a, b, d$. Hence, the one-dimensional case (Lemma B.8) implies

$\int_{-1+a x_{2}}^{1+b x_{2}} \omega^{1 / 2}\left(x_{1}, x_{2}\right) v^{2}\left(x_{1}, x_{2}\right) d x_{1} \leq C(p+1)^{2} \int_{-1+a x_{2}}^{1+b x_{2}} \sqrt{\omega\left(x_{1}, x_{2}\right) \Phi_{a, b, d}\left(x_{1}, x_{2}\right)} v^{2}\left(x_{1}, x_{2}\right) d x_{1}$.

Integrating this estimate over $x_{2} \in(0, d)$ completes the proof.

Proof of Lemma B.7. By using an affine pullback we may restrict to the case that $K$ is the equi-sided triangle conv $\left(\left(\begin{array}{l}0 \\ 0\end{array}\right),\left(\begin{array}{l}1 \\ 0\end{array}\right), \frac{1}{2}\left(\begin{array}{c}1 \\ \sqrt{3}\end{array}\right)\right)$ and $E=(0,1) \times\{0\}$.

It turns out that the proofs for the different cases in (6.4) for $\varphi_{E}$ and in (5.4) for $\Phi_{K}$ uses the same arguments and we work them out exemplarily for the case of the quadratic edge bubble

$$
\varphi_{E}\left(x_{1}, x_{2}\right)=\left(x_{1}-\frac{x_{2}}{\sqrt{3}}\right)\left(1-x_{1}-\frac{x_{2}}{\sqrt{3}}\right)
$$

and for $\Phi_{K}=\Phi_{K}^{(3)}$ being the cubic bubble on $K$.

First, we will cover $K$ with 4 trapezoids and one triangle: Let $v=\left(\cos \frac{\pi}{4}, \sin \frac{\pi}{4}\right)^{\top}=$ $2^{-1 / 2}(1,1)^{\top}$. Then,

1. $T_{1}:=\left\{\left(\begin{array}{c}\hat{x}_{1} \\ 0\end{array}\right)+s v:\left(\begin{array}{l}0 \leq \hat{x}_{1} \leq 1 / 2 \\ 0 \leq s \leq L_{1}\left(\hat{x}_{1}\right)\end{array}\right)\right\}$ with $L_{1}\left(\hat{x}_{1}\right):=\frac{\sqrt{6}}{1+\sqrt{3}}\left(1-\hat{x}_{1}\right)$.

2. $T_{2}$ : mirror image of $T_{1}$ with respect to the angle bisector at $(0,0)^{\top}$.

3. $T_{3}$ : counter-clockwise rotations of $T_{1}$ by $\frac{3 \pi}{4}$ about the barycenter of $K$.

4. $T_{4}$ : mirror image of $T_{3}$ with respect to the angle bisector at $(1,0)^{\top}$.

5. $T_{5}:=\left\{\left(x_{1}, x_{2}\right)^{\top} \in K \mid x_{2} \geq 1 / 2\right\}$.

Case $T_{1}$ : We introduce

$$
\chi:\left(\begin{array}{c}
0 \leq \hat{x}_{1} \leq 1 / 2 \\
0 \leq s \leq L_{1}\left(\hat{x}_{1}\right)
\end{array}\right) \rightarrow T_{1} \quad \text { by } \quad \chi\left(\hat{x}_{1}, s\right):=\left(\begin{array}{c}
\hat{x}_{1} \\
0
\end{array}\right)+s v
$$

The bubble function $\varphi_{E}$ restricted to the line $\left(\begin{array}{c}\hat{x}_{1} \\ 0\end{array}\right)+s v$ results in

$$
\psi_{\hat{x}_{1}}(s):=\varphi_{E} \circ \chi\left(\hat{x}_{1}, s\right)=\left(L_{1}\left(\hat{x}_{1}\right)-s\right)\left(\frac{\sqrt{3}+1}{\sqrt{6}} \hat{x}_{1}+\frac{s}{3}\right) \quad \forall\left(\begin{array}{l}
0 \leq \hat{x}_{1} \leq 1 / 2 \\
0 \leq s \leq L_{1}\left(\hat{x}_{1}\right)
\end{array}\right) .
$$

Note that the function $\psi_{\hat{x}_{1}}$ satisfies the assumptions of Lemma B.8 and $\hat{v}:=v \circ \chi$ is a polynomial of maximal degree $p$. Hence,

$$
\begin{aligned}
\int_{T_{1}} \varphi_{E} v^{2} & =\int_{0}^{1 / 2}\left(\int_{0}^{L_{1}\left(\hat{x}_{1}\right)} \psi_{\hat{x}_{1}}(s) \hat{v}^{2} d s\right) d x_{1} \\
& \leq C(p+1)^{2} \int_{0}^{1 / 2}\left(\int_{0}^{L_{1}\left(\hat{x}_{1}\right)} \psi_{\hat{x}_{1}}(s) \Phi_{\left[0, L_{1}\left(\hat{x}_{1}\right)\right]}(s) \hat{v}^{2} d s\right) d \hat{x}_{1} .
\end{aligned}
$$


Composing $\Phi_{\left[0, L_{1}\left(\hat{x}_{1}\right)\right]}(s)$ with $\chi^{-1}$ yields the function

$$
d\left(x_{1}, x_{2}\right)=\frac{1+\sqrt{3}}{\sqrt{3}} \frac{x_{2}\left(1-x_{1}-\frac{x_{2}}{\sqrt{3}}\right)}{\left(1-x_{1}+x_{2}\right)^{2}} .
$$

Note that the distance function $\Phi_{K}^{1}\left(x_{1}, x_{2}\right)=\operatorname{dist}\left(\left(x_{1}, x_{2}\right)^{\top}, \partial K\right)$ is piecewise linear on $K$. It is easy to verify that $d\left(x_{1}, x_{2}\right) \leq C \Phi_{K}^{1}\left(x_{1}, x_{2}\right)$ pointwise on $T_{1}$ for some $C=O(1)$ so that

$$
\int_{T_{1}} \varphi_{E} v^{2} \leq C^{\prime}(p+1)^{2} \int_{T_{1}} \varphi_{E} \Phi_{K}^{1} v^{2}
$$

Since $\varphi_{E} \Phi_{K}^{1} \leq \tilde{C} \Phi_{K}$ pointwise on $K$ we have proved the assertion for $T_{1}$.

Case $T_{3}$ : The proof for the trapezoid $T_{3}$ follows by symmetry.

Case $T_{2}$ : Next, we will consider the trapezoid $T_{2}$ and first note that by interchanging the $x_{1}, x_{2}$-variables the case becomes equivalent to the estimate

$$
\int_{T_{1}} \varphi_{\tilde{E}} v^{2} \leq C^{\prime}(p+1)^{2} \int_{T_{1}} \Phi_{K}^{(3)} v^{2} \quad v \in \mathbb{P}_{p}\left(T_{1}\right)
$$

where $\varphi_{\tilde{E}}$ is the qudratic edge bubble for the edge $\tilde{E}=\overline{\left(\begin{array}{l}0 \\ 0\end{array}\right),\left(\begin{array}{c}1 / 2 \\ \sqrt{3} / 2\end{array}\right)}$ with explicit form

$$
\varphi_{\tilde{E}}\left(x_{1}, x_{2}\right)=\frac{2}{\sqrt{3}} x_{2}\left(1-x_{1}-\frac{x_{2}}{\sqrt{3}}\right)
$$

This time, the bubble function $\varphi_{\tilde{E}}$, restricted to the line $\left(\begin{array}{c}\hat{x}_{1} \\ 0\end{array}\right)+s v$, is given by

$$
\tilde{\psi}_{\hat{x}_{1}}(s):=\varphi_{\tilde{E}} \circ \chi\left(\hat{x}_{1}, s\right)=\frac{\sqrt{3}+1}{3} s\left(L\left(\hat{x}_{1}\right)-s\right) \quad \forall\left(\begin{array}{l}
0 \leq \hat{x}_{1} \leq 1 / 2 \\
0 \leq s \leq L_{1}\left(\hat{x}_{1}\right)
\end{array}\right) .
$$

The function $\tilde{\psi}_{\hat{x}_{1}}$ satisfies the assumptions of Lemma B.8 so that

$$
\begin{aligned}
\int_{T_{1}} \varphi_{\tilde{E}} v^{2} & =\int_{0}^{1 / 2}\left(\int_{0}^{L_{1}\left(\hat{x}_{1}\right)} \tilde{\psi}_{\hat{x}_{1}}(s) \hat{v}^{2} d s\right) d x_{1} \\
& \leq C(p+1)^{2} \int_{0}^{1 / 2}\left(\int_{0}^{L_{1}\left(\hat{x}_{1}\right)} \tilde{\psi}_{\hat{x}_{1}}(s) \Phi_{\left[0, L_{1}\left(\hat{x}_{1}\right)\right]}(s) \hat{v}^{2} d s\right) d \hat{x}_{1} .
\end{aligned}
$$

Now we can argue as for the Case of $T_{1}$ to obtain

$$
\int_{T_{1}} \varphi_{\tilde{E}} v^{2} \leq C^{\prime}(p+1)^{2} \int_{T_{1}} \varphi_{\tilde{E}} \Phi_{K}^{1} v^{2}
$$

Since $\varphi_{\tilde{E}} \Phi_{K}^{1} \leq \tilde{C} \Phi_{K}$ pointwise on $K$ the assertion follows for $T_{2}$.

Case $T_{4}$ : The proof for the trapezoid $T_{4}$ again follows by symmetry from the case $T_{2}$.

Case $T_{5}$ : On $T_{5}$ we have the pointwise estimate $\varphi_{E} \leq C \Phi_{K}^{(3)}$ and the estimate for $T_{5}$ is trivial. 


\section{References}

[1] M. Ainsworth and J. T. Oden. A Posteriori Error Estimation in Finite Element Analysis. Wiley, 2000.

[2] I. Babuška and W. C. Rheinboldt. A-posteriori error estimates for the finite element method. Internat. J. Numer. Meth. Engrg., 12:1597-1615, 1978.

[3] I. Babuška and W. C. Rheinboldt. Error estimates for adaptive finite element computations. SIAM J. Numer. Anal., 15:736-754, 1978.

[4] W. Bangerth and R. Rannacher. Adaptive Finite Element Methods for Differential Equations. Birkhäuser, Basel, 2003.

[5] R. E. Bank and A. Weiser. Some a posteriori error estimators for elliptic partial differential equations. Math. Comp., 44:283-301, 1985.

[6] R. E. Bank and J. Xu. Asymptotically exact a posteriori error estimators. II. General unstructured grids. SIAM J. Numer. Anal., 41(6):2313-2332, 2003.

[7] P. Binev, W. Dahmen, and R. DeVore. Adaptive finite element methods with convergence rates. Numer. Math., 97(2):219-268, 2004.

[8] F. A. Bornemann, B. Erdmann, and R. Kornhuber. A posteriori error estimates for elliptic problems in two and three space dimensions. SIAM J. Numer. Anal., 33(3):1188-1204, 1996.

[9] P. Ciarlet. The finite element method for elliptic problems. North-Holland, 1987.

[10] W. Dörfler. A convergent adaptive algorithm for Poisson's equation. SIAM J. Numer. Anal., 33(3):1106-1124, 1996.

[11] W. Dörfler and R. H. Nochetto. Small data oscillation implies the saturation assumption. Numer. Math., 91(1):1-12, 2002.

[12] T. Koornwinder. Two-variable analogues of the classical orthogonal polynomials. In Theory and application of special functions (Proc. Advanced Sem., Math. Res. Center, Univ. Wisconsin, Madison, Wis., 1975), pages 435-495. Math. Res. Center, Univ. Wisconsin, Publ. No. 35. Academic Press, New York, 1975.

[13] T. H. Koornwinder and S. A. Sauter. The intersection of bivariate orthogonal polynomials on triangle patches. Math.Comp., in press. arXiv:1307.8429v3 [math.NA].

[14] K. Mekchay and R. H. Nochetto. Convergence of adaptive finite element methods for general second order linear elliptic PDEs. SIAM J. Numer. Anal., 43(5):1803-1827, 2005.

[15] J. M. Melenk. $h p$-interpolation of nonsmooth functions and an application to $h p$-a posteriori error estimation. SIAM J. Numer. Anal., 43(1):127-155, 2005.

[16] J. M. Melenk and B. I. Wohlmuth. On residual-based a posteriori error estimation in hp-FEM. Adv. Comput. Math., 15(1-4):311-331 (2002), 2001. 
[17] M. Melenk. HP-interpolation of non-smooth functions (extended version). Technical Report NI03050, Isaac Newton Institute for Mathematical Sciences, 2003.

[18] J. Proriol. Sur une famille de polynomes à deux variables orthogonaux dans un triangle. C. R. Acad. Sci. Paris, 245:2459-2461, 1957.

[19] S. Repin. A posteriori estimates for partial differential equations, volume 4. Walter de Gruyter GmbH \& Co. KG, Berlin, 2008.

[20] R. Stevenson. An optimal adaptive finite element method. SIAM J. Numer. Anal., 42(5):2188-2217, 2005.

[21] R. Stevenson. Optimality of a standard adaptive finite element method. Found. Comput. Math., 7(2):245-269, 2007.

[22] R. Verfürth. A review of a posteriori error estimation and adaptive mesh refinement. Wiley and Teubner, 1996.

[23] R. Verfürth. A posteriori error estimation techniques for finite element methods. Oxford University Press, Oxford, 2013. 